\title{
Transcriptome and Small RNA Combined Sequencing Analysis of Cold Tolerance in Non-heading Chinese Cabbage
}

\author{
Jin Wang 1,2, Qinxue Zhang ${ }^{1}$, Xiong You ${ }^{3 *}$ and Xilin Hou ${ }^{1 *}$ \\ ${ }^{1}$ State Key Laboratory of Crop Genetics and Germplasm Enhancement/Key Laboratory of Biology and Germplasm \\ Enhancement of Horticultural Crops in East China, Ministry of Agriculture/Engineering Research Center of Germplasm \\ Enhancement and Utilization of Horticultural Crops, Ministry of Education, Nanjing Agricultural University, Nanjing, China, \\ ${ }^{2}$ School of Life Sciences, Jiangsu University, Zhenjiang, China, ${ }^{3}$ College of Sciences, Nanjing Agricultural University, Nanjing, \\ China
}

Background: Non-heading Chinese cabbage (Brassica rapa ssp. chinensis) is an important leaf vegetable grown worldwide. However, there has currently been not enough transcriptome and small RNA combined sequencing analysis of cold tolerance, which hinders further functional genomics research.

OPEN ACCESS

Edited by:

Abdelfattah Badr

Helwan University, Egypt

Reviewed by:

Arif Ashraf,

University of Massachusetts Amherst,

United States

Jianjun Zhao,

Agricultural University of Hebei, China

${ }^{*}$ Correspondence: Xiong You

youx@njau.edu.cn

Xilin Hou

hxl@njau.edu.cn

Specialty section:

This article was submitted to

Plant Genomics,

a section of the journal

Frontiers in Genetics

Received: 11 September 2020

Accepted: 29 March 2021

Published: 21 July 2021

Citation:

Wang J, Zhang Q, You X and Hou X (2021) Transcriptome

and Small RNA Combined

Sequencing Analysis of Cold

Tolerance in Non-heading Chinese

Cabbage. Front. Genet. 12:605292.

doi: 10.3389/fgene.2021.605292
Results: In this study, $63.43 \mathrm{~Gb}$ of clean data was obtained from the transcriptome analysis. The clean data of each sample reached $6.99 \mathrm{~Gb}$, and the basic percentage of Q30 was 93.68\% and above. The clean reads of each sample were sequence aligned with the designated reference genome (Brassica rapa, IVFCAASv1), and the efficiency of the alignment varied from 81.54 to $87.24 \%$. According to the comparison results, 1,860 new genes were discovered in Pak-choi, of which 1,613 were functionally annotated. Among them, 13 common differentially expressed genes were detected in all materials, including seven upregulated and six downregulated. At the same time, we used quantitative real-time PCR to confirm the changes of these gene expression levels. In addition, we sequenced miRNA of the same material. Our findings revealed a total of 34,182,333 small RNA reads, 88,604,604 kinds of small RNAs, among which the most common size was $24 \mathrm{nt}$. In all materials, the number of common differential miRNAs is eight. According to the corresponding relationship between miRNA and its target genes, we carried out Gene Ontology and Kyoto Encyclopedia of Genes and Genomes enrichment analysis on the set of target genes on each group of differentially expressed miRNAs. Through the analysis, it is found that the distributions of candidate target genes in different materials are different. We not only used transcriptome sequencing and small RNA sequencing but also used experiments to prove the expression levels of differentially expressed genes that were obtained by sequencing. Sequencing combined with experiments proved the mechanism of some differential gene expression levels after low-temperature treatment.

Conclusion: In all, this study provides a resource for genetic and genomic research under abiotic stress in Pak-choi.

Keywords: non-heading Chinese cabbage, transcriptome sequencing, small RNA sequencing, cold tolerance, expression pattern 


\section{BACKGROUND}

Non-heading Chinese cabbage (Brassica rapa ssp. chinensis), also known as Pak-choi, is one of the most common cultivated vegetables in China. Its growth and productivity are often adversely affected by cold and even freezing stresses from the environment (Abe et al., 2003; Yu et al., 2010; Li et al., 2016). The response mechanism of Arabidopsis' cold acclimation and freezing resistance is not exactly the same (Rahman et al., 2020). The cold domestication of plants, which usually involves many biochemical and physiological changes, is complicated and difficult to understand (Salinas, 2002; Kaplan et al., 2004; Chinnusamy et al., 2007; Krasensky and Jonak, 2012). In Arabidopsis, the latest study found that the cold stress response was mediated by the GNOM ARF-GEF pathway (Ashraf and Rahman, 2019). After low-temperature treatment, the metabolism and transcriptome of plants are greatly affected, and the expression levels of certain genes are also regulated. Some related metabolic enzymes are inhibited, and the degree of plant metabolism are affected to some extent (Chinnusamy et al., 2007). Nowadays, genetic, biochemical, and various sequencing methods have been applied to detect how some genes cope with environmental stresses (Chinnusamy et al., 2007; Krasensky and Jonak, 2012), and many plants have been studied today, such as rice (Hussain et al., 2016; Wang et al., 2016), cotton (Zhao et al., 2012), tomato (Starck et al., 2000), potato (Shinozaki and Yamaguchi-Shinozaki, 1996), muskmelons (Wang et al., 2004), and sugarcane (Thakur et al., 2010; Anjum et al., 2011; Zhu et al., 2013). However, the response mechanism of non-heading Chinese cabbage to cold stress remains unclear, and further joint research is needed.

Nowadays, transcriptome analysis has gradually become a useful and general tool for discovering genes in multiple stress pathways, including determining the expression patterns of related genes (Martin and Wang, 2011; Hamilton and Robin Buell, 2012; Ward et al., 2012; Li et al., 2020). The related genes reported in plant secondary metabolism were discovered based on experimental methods of functional genome sequencing (Dixon, 2001; Goossens et al., 2003). In addition, RNA sequencing technology is often used to obtain complete transcriptome information from different plants, such as tea tree, chlorophytum borivilianum, and atractylodes lancea, and provide better insights into transcription or posttranscriptional force, including regulation of essential genes, during secondary metabolite biosynthetic pathways (Kalra et al., 2013; Li et al., 2015; Devi et al., 2016). Nowadays, transcriptome sequencing has been successfully used to detect expression levels of related genes in many organisms, such as rice (Zhang et al., 2010), yeast (Nagalakshmi et al., 2008; Wilhelm et al., 2008), sweet potato (Wang et al., 2010), and taxus (Ge et al., 2011). With Illumina sequencing technology, millions of sequences were

Abbreviations: COG, Cluster of Orthologus Groups; CYP1, Cyclophilin 1; DEGs, Differentially expressed genes; FC, Fold change; FDR, False discovery rate; GO, Gene ontology; hc-siRNA, heterochromatic short interfering RNA; KEGG, Kyoto Encyclopedia of Genes and Genomes; miRNA, microRNA; nt, nucleotides; qRT-PCR, quantitative real-time polymerase chain reaction; RNA-Seq, RNA sequencing; ta-siRNA, trans short interfering RNA. read at a time, and individual assembled genes were mapped into a reference transcriptome map for molecular annotation (Cheng et al., 2015).

Studies have found that microRNA (miRNA), trans short interfering RNA (ta-siRNA), and heterochromatic short interfering RNA (hc-siRNA), all play important roles in different organisms (Axtell, 2013). Among them, miRNA, a type of endogenous small RNA, is composed of about 22 nucleotides (nt) and usually plays a negative role in regulating gene expression (Voinnet, 2009). Many studies have shown that miRNAs are often involved in plant development, hormone signaling, and abiotic stress responses (Jones-Rhoades et al., 2006; Chambers and Shuai, 2009). Generally speaking, small interfering RNAs are processed from perfect double-stranded RNA, while miRNAs are derived from single-stranded RNA transcripts, forming an imperfect double-stranded stem-loop precursor structure (Llave et al., 2002; Khraiwesh et al., 2010; Hao et al., 2012; Szittya and Burgyán, 2013). On the whole, miRNA plays a vital role in various biological and metabolic processes of plant growth and development, such as biotic (or abiotic) stresses, which can also negatively regulate the expression of target genes, by inhibiting (cutting) target mRNA or other ways (Bartel, 2004; Jones-Rhoades and Bartel, 2004; Jones-Rhoades et al., 2006; Mallory and Vaucheret, 2006; Sunkar et al., 2006; Voinnet, 2009; Wu et al., 2010). Therefore, it is important to identify miRNAs and their target genes (or miRNAs), which is essential for a better understanding of miRNA-mediated regulation of cold stress genes.

In this study, we compared the tolerance of two common and typical non-heading Chinese cabbage varieties, Suzhouqing (BcL.1) and Sijiucaixin (BcL.2), to cold stress. We found that $B c L .1$ is more tolerant to cold stress compared with BcL.2. We used RNA-Seq for comprehensive characterization and explored the effects of low temperature. We identified the most important genes in the low-temperature response and discussed their regulatory networks under cold stress. Furthermore, we identified conserved and novel miRNAs and their potential target genes in non-heading Chinese cabbage, and discussed the possible connections between them. Quantitative real-time polymerase chain reaction (qRT-PCR) was also used to assess the expression levels of common differentially expressed genes (DEGs) and identify those candidate genes involved in cold tolerance. This work might serve as a reference of the functional analysis of cold tolerance in non-heading Chinese cabbage.

\section{RESULTS}

\section{Quantity Statistics and Venn Diagram of Differentially Expressed Genes}

To study the effects of temperature on plant growth, plants (BcL.1 and BcL.2) were grown for $6 \mathrm{~h}$ in environments of 25 and $4^{\circ} \mathrm{C}$. Except for the temperature, the other conditions remain unvaried. Then, we observed that low temperatures have an important effect on plant phenotype. Whether it is BcL.1 or BcL.2, under $4^{\circ} \mathrm{C}$ treatment, plant leaves are more likely to shrink or even wither than under the $25^{\circ} \mathrm{C}$ treatment (Figure 1). This 

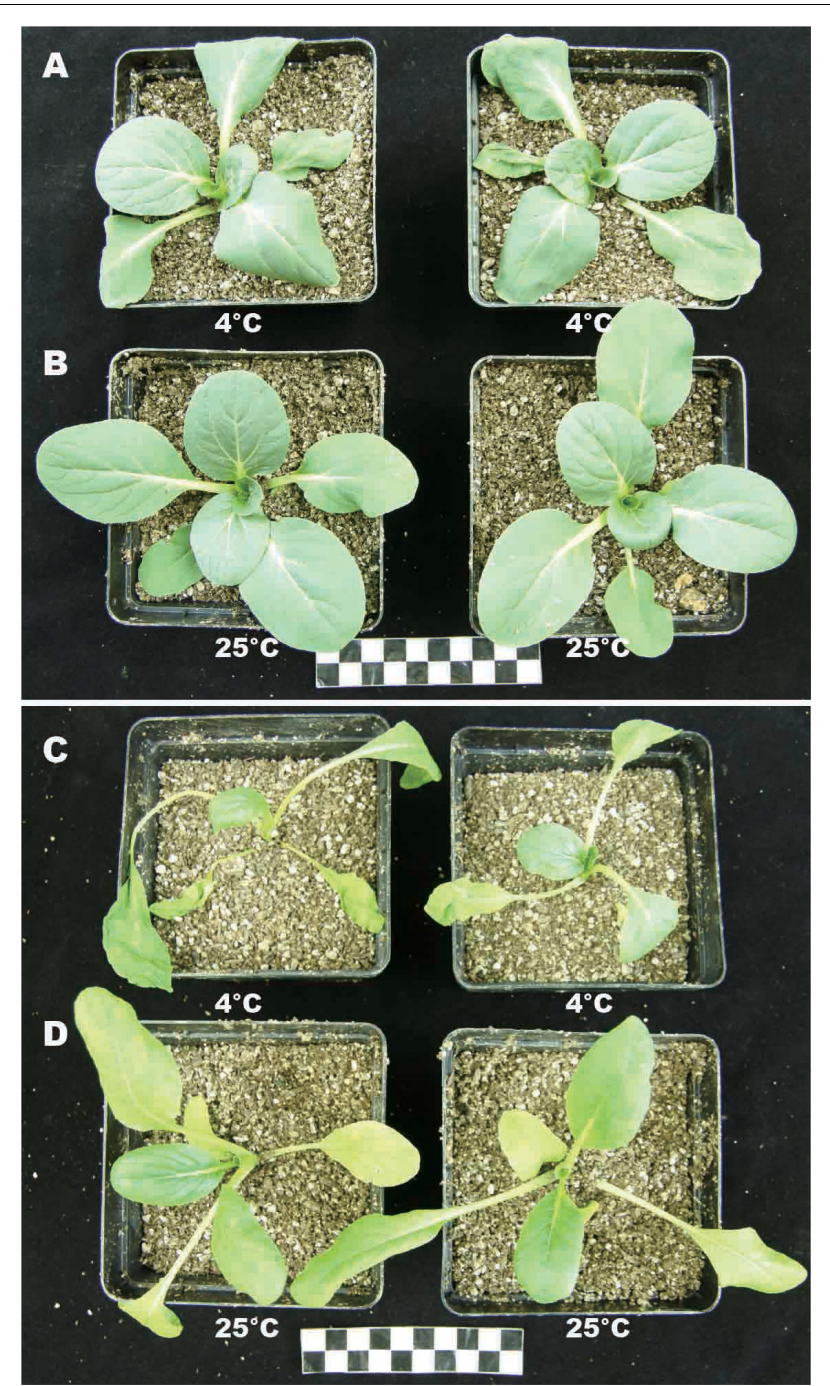

FIGURE 1 | Phenotype of BCL. 1 and BCL.2 under cold stress. Approximately 30-day-old seedlings were subjected to cold stress $\left(4^{\circ} \mathrm{C}\right.$ for $\left.6 \mathrm{~h}\right)$; plants grown under normal condition $\left(22^{\circ} \mathrm{C}\right)$ for $6 \mathrm{~h}$ were used as control. (A) BCL. 1-4. (B) BCL.1-25. (C) BCL.2-4. (D) BCL.2-25.

result corresponds to previous reports that cold stress usually downgrades the seedling vigor (Kang and Saltveit, 2002; Wang et al., 2016) and causes leaf atrophy, slows crop growth, and ultimately reduces the yield (Cruz and Milach, 2004; Oliver et al., 2007; Ruelland et al., 2009).

Afterward, to study the upregulation and downregulation of common genes shared by each group of treatments, we established a Venn diagram of differentially expressed genes. Between the G0 (BcL.2-25 vs. BcL.2-4) and G2 (BcL.1-25 vs. $B c L .1-4)$ groups, a total of 313 common genes were upregulated, and 308 common genes were downregulated (Figures 2A,B). Meanwhile, between the G1 (BcL.1-25 vs. BcL.2-25) and G3 (BcL.1-4 vs. BcL.2-4) groups, a total of 344 common genes were found to be upregulated, and 117 common genes were downregulated (Figures 2C,D). In all materials, a total of seven common genes were upregulated, and six common genes were downregulated (Figures 2E,F and Supplementary Table 1). We speculated that these DEGs might help increase the potential application value of non-heading Chinese cabbage under cold stress.

\section{Functional Annotation and Classification}

Between the BcL.1-25 and BcL.1-4 groups, 6,208 DEGs $(p<0.05)$ were detected, including 3,639 upregulated and 2,569 downregulated genes (Figure 3C). The annotated unigenes were then assigned to Gene Ontology (GO) terms for functional classification. Three main categories (biological process, molecular function, and cellular component) of GO classification were analyzed separately to investigate their functional distribution. To simplify the functional distribution of plants, we assigned the annotated sequences to GO-slim terms to obtain a "thin" version of classification (Gao et al., 2015). Cellular process (GO:0009987, 3,589 genes) and metabolic process (GO:0008152, 3,424 genes) in the biological process, cell part (GO:0004464, 5,170 genes) and cells (GO:0005623, 5,169 genes) in the cellular component and binding activity (GO:0005488, 2,808 genes), and catalytic activity (GO:0003824, 2,279 genes) in the molecular function were the most representative level 2 GO terms in all three data sets, respectively (Figure $\mathbf{3 A}$ and Supplementary Table 2). To further identify the active biochemical pathways, we mapped it to the reference canonical pathways in the Kyoto Encyclopedia of Genes and Genomes (KEGG). KEGG is thought to provide a basic platform for systematic analysis of gene function in terms of the network of gene products (Kanehisa and Goto, 2000). A total of 24,199 unigenes were annotated based on a BLASTX search of the KEGG database (Supplementary Table 3): 263 biosynthesis pathways were predicted and classified into five categories, of which the ribosome pathway was the largest, containing 287 genes (287 out of 1,586, 18.10\%) (Figure 3B, Supplementary Figure 1A, and Supplementary Table 10). The annotated unigenes were categorized into different functional groups based on the Cluster of Orthologus Groups (COG) database (Supplementary Table 14). Unigenes $(3,700)$ could be classified into 23 COG categories. Out of the 3,700 unigenes, general function prediction only $(679,18.35 \%)$ was assigned to the COG category of general function prediction, which represented the largest functional group of the 23 COG categories, followed by translation, ribosomal structure, and biogenesis $(433,11.70 \%)$, transcription (329, 8.90\%), replication, recombination and repair (293, $7.92 \%)$, and signal transduction mechanisms (290, 7.84\%) (Supplementary Figure 2A).

Between the BcL.2-25 and BcL.2-4 groups, 964 DEGs $(p<0.05)$ were detected, including 437 upregulated and 527 downregulated genes (Figure 4C). Through GO enrichment stratification analysis, cellular process (GO:0009987, 552 genes) and metabolic process (GO:0008152, 534 genes) in the biological process, cell part (GO:0004464, 741 genes) and cells (GO:0005623, 741 genes) in the cellular component and binding activity (GO:0005488, 429 genes), and catalytic activity (GO:0003824, 433 genes) in the molecular function 

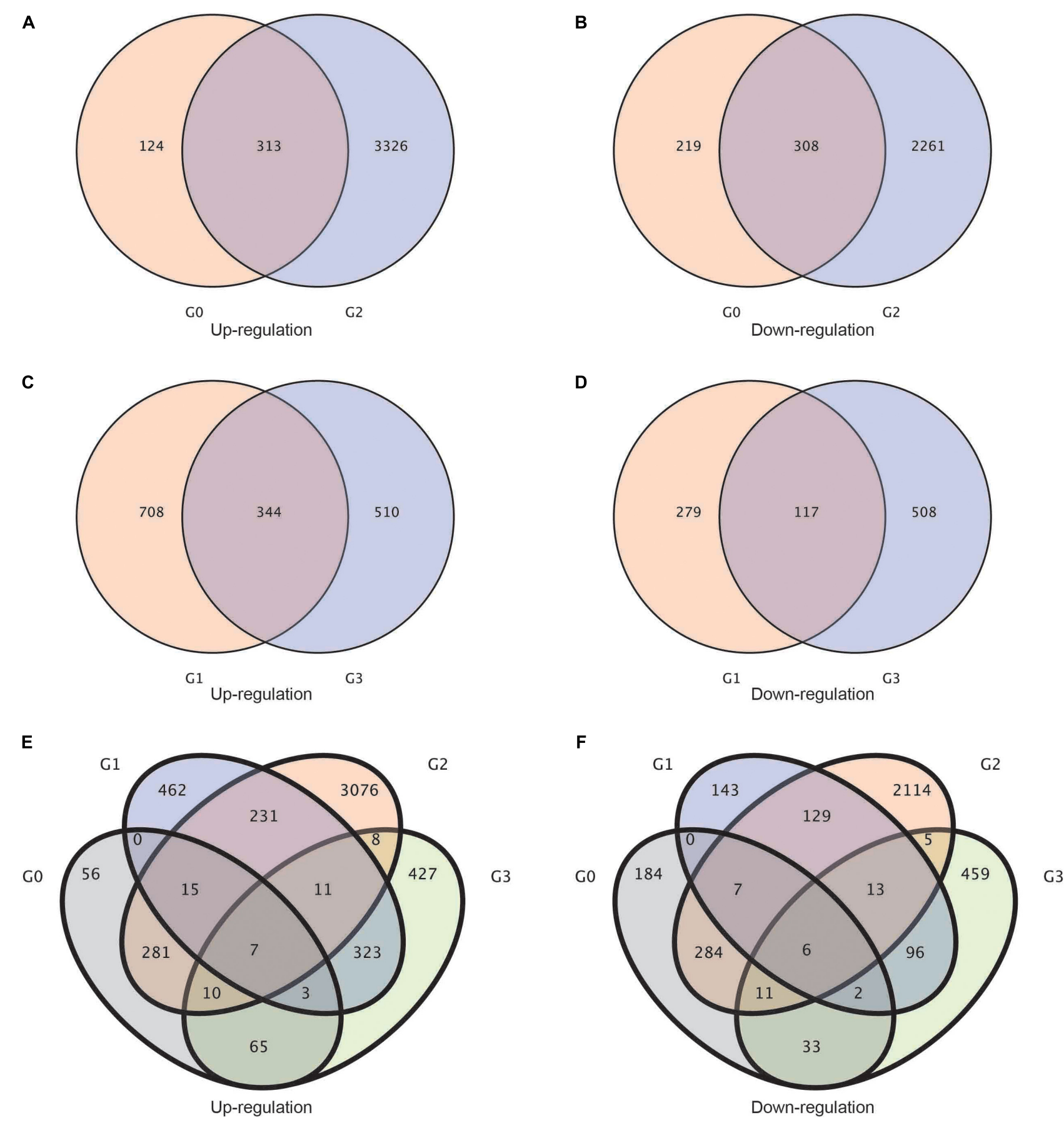

FIGURE 2 | Statistics of the common genes and specific genes in different groups. (A,B) Venn diagram of up-/downregulated differentially expressed genes between G2 and G0 groups. (C,D) Venn diagram of up-/downregulated differentially expressed genes between G3 and G1 groups. (E,F) Venn diagram of up/downregulated differentially expressed genes in all groups. G0: BCL.2-25 vs. BCL.2-4; G1: BCL.1-25 vs. BCL.2-25; G2: BCL.1-25 vs. BCL.1-4; G3: BCL.1-4 vs. BCL.2-4.

were the most representative level 2 GO terms, respectively (Figure 4A and Supplementary Table 4). Through KEGG pathway enrichment analysis (Supplementary Table 5), the protein processing in the endoplasmic reticulum pathway was the largest, containing 26 genes (26 out of 251, 10.36\%) (Figure 4B, Supplementary Figure 1B, and Supplementary
Table 11). Through COG classification of differentially expressed genes, out of 554 unigenes (Supplementary Table 15), general function prediction only $(109,19.68 \%)$ was assigned to the COG category of general function prediction, which represented the largest functional group of the $21 \mathrm{COG}$ categories, followed by posttranslational modification, protein 


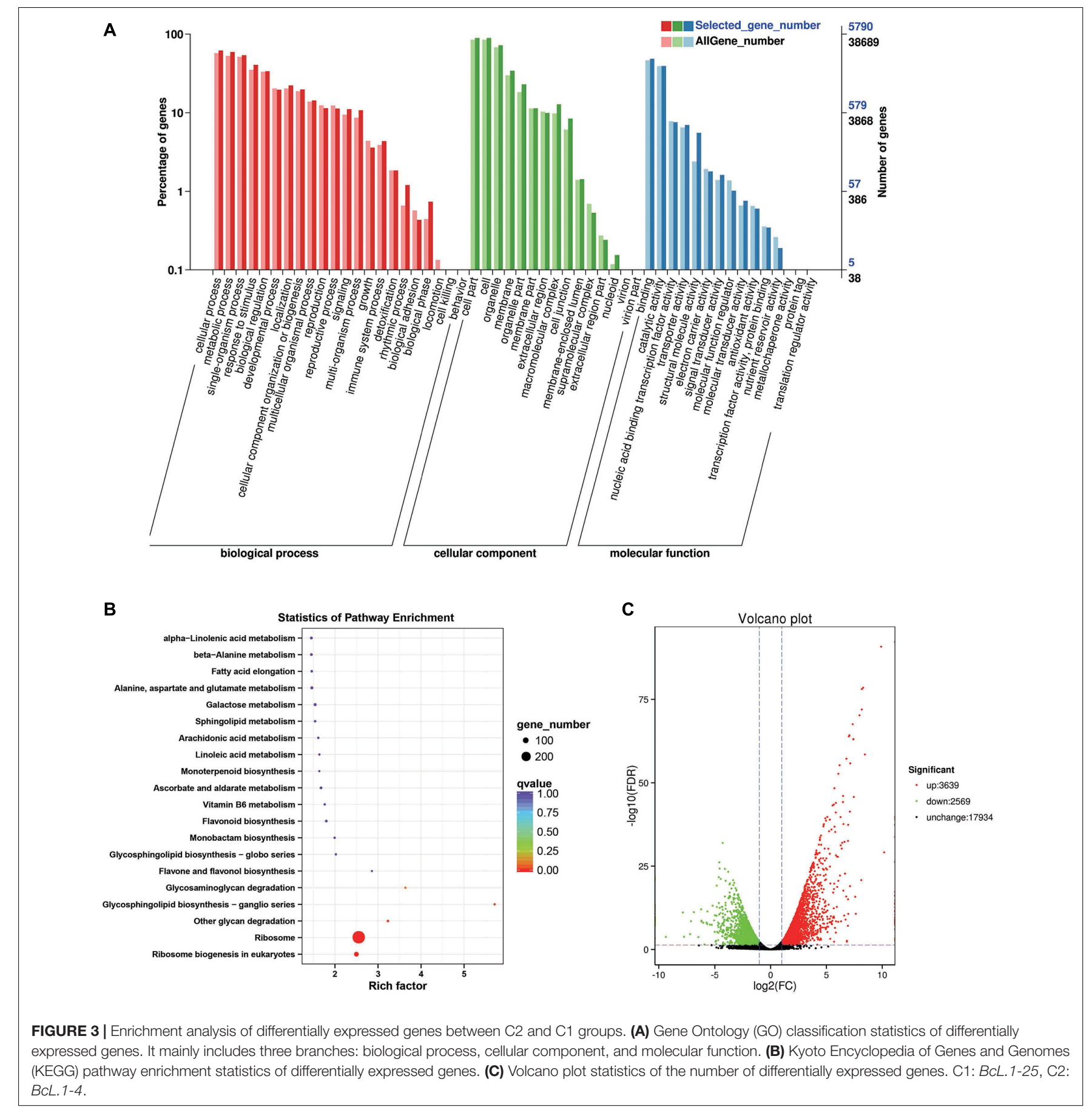

turnover, chaperones $(59,10.65 \%)$, amino acid transport and metabolism $(49,8.84 \%)$, carbohydrate transport and metabolism $(45,8.12 \%)$, and signal transduction mechanisms $(35,6.32 \%)$ (Supplementary Figure 2B).

Between the BcL.1-25 and BcL.2-25 groups, 1,448 DEGs $(p<0.05)$ were detected, including 1,052 upregulated and 396 downregulated genes (Figure 5C). Through GO enrichment stratification analysis, cellular process (GO:0009987, 729 genes) and metabolic process (GO:0008152, 695 genes) in the biological process, cell part (GO:0004464, 988 genes) and cells (GO:0005623, 989 genes) in the cellular component and binding activity (GO:0005488, 556 genes), and catalytic activity (GO:0003824, 465 genes) in the molecular function were the most representative level 2 GO terms, respectively (Figure 5A and Supplementary Table 6). Through KEGG pathway enrichment analysis (Supplementary Table 7), the ribosome pathway was the largest, containing 96 genes (96 out of 366, 26.23\%) (Figure 5B, Supplementary Figure 1C, and Supplementary Table 12). 


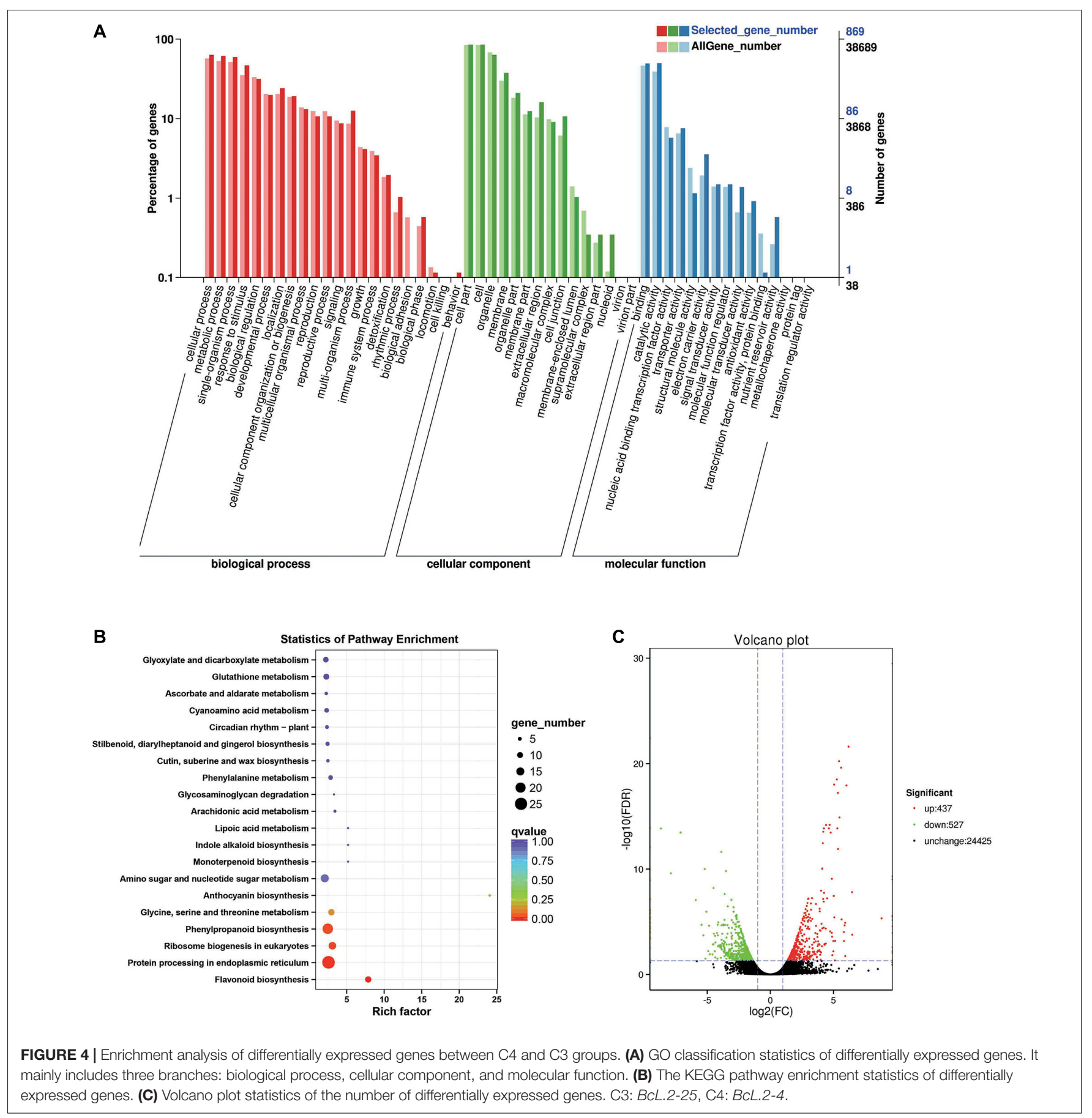

Through COG classification of differentially expressed genes, out of 681 unigenes (Supplementary Table 16), general function prediction only $(124,18.21 \%)$ was assigned to the COG category of general function prediction, which represented the largest functional group of the 23 COG categories, followed by translation, ribosomal structure, and biogenesis (109, 16.01\%), transcription $(51,7.49 \%)$, replication, recombination, and repair (50, 7.34\%), and amino acid transport and metabolism (44, 6.46\%) (Supplementary Figure 2C).
Between the BcL.1-4 and BcL.2-4 groups, 1,479 DEGs $(p<0.05)$ were detected, including 854 upregulated and 625 downregulated genes (Figure 6C). Through GO enrichment stratification analysis, cellular process (GO:0009987, 750 genes) and metabolic process (GO:0008152, 697 genes) in the biological process, cell part (GO:0004464, 1,056 genes) and cells (GO:0005623, 1,057 genes) in the cellular component and binding (GO:0005488, 610 genes), and catalytic activity (GO:0003824, 536 genes) in the molecular 

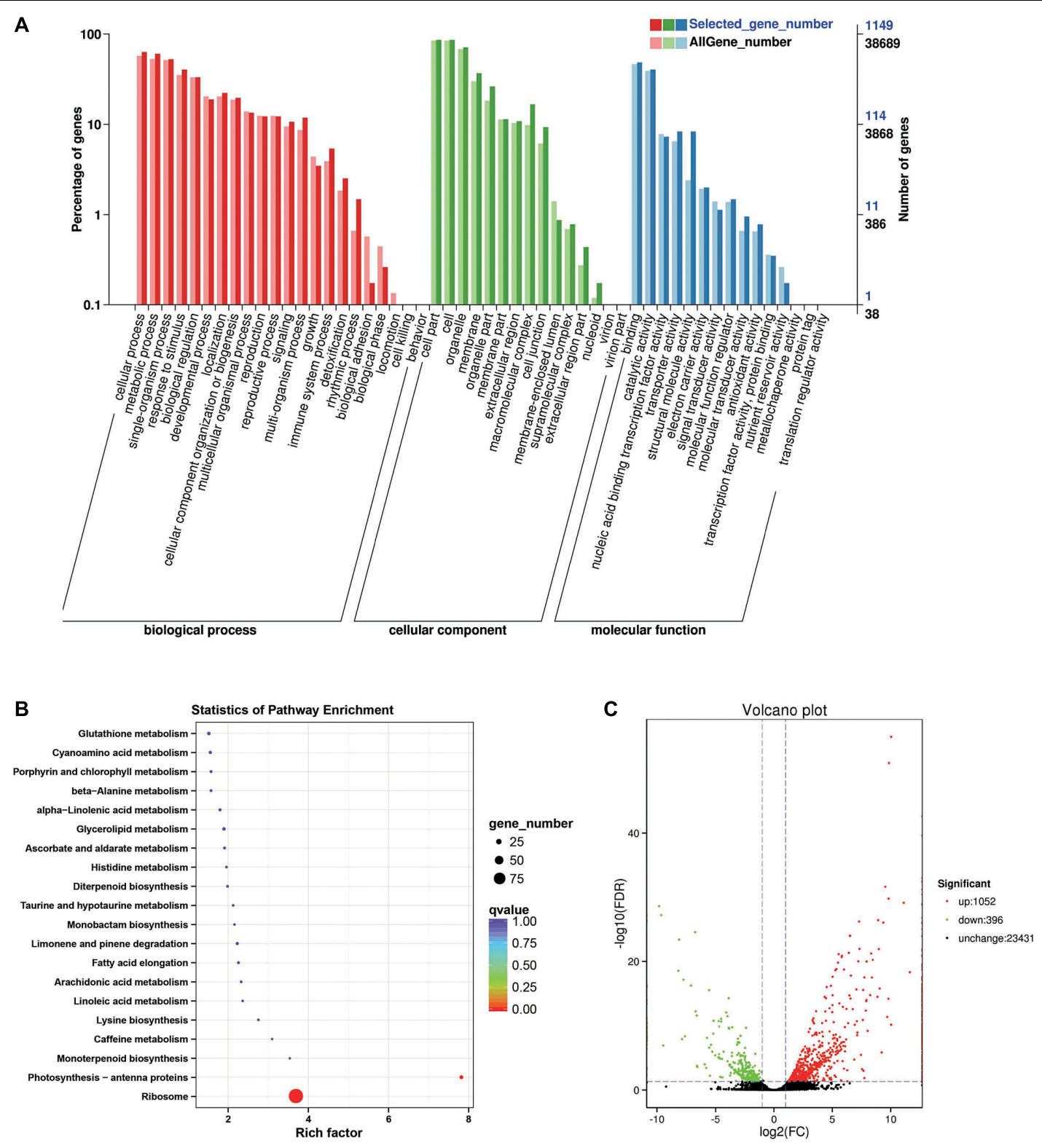

FIGURE 5 | Enrichment analysis of differentially expressed genes between C3 and C1 groups. (A) GO classification statistics of differentially expressed genes. It mainly includes three branches: biological process, cellular component, and molecular function. (B) KEGG pathway enrichment statistics of differentially expressed genes. (C) Volcano plot statistics of the number of differentially expressed genes. C1: BCL.1-25, C3: BCL.2-25.

function were the most representative level 2 GO terms, respectively (Figure 6A and Supplementary Table 8). Through the KEGG pathway enrichment analysis (Supplementary Table S9), the DNA replication pathway was the largest, containing 13 genes (13 out of 329, 3.95\%) (Figure 6B, Supplementary Figure 1D, and Supplementary Table 13). Through COG classification of differentially expressed genes, out of 681 unigenes (Supplementary Table 17), general function prediction only $(144,17.98 \%)$ was assigned into the COG category of general function prediction, which represented the largest functional group of the 23 COG categories, followed by carbohydrate transport and metabolism
(74, 9.24\%), posttranslational modification, protein turnover, chaperones $(67,8.36 \%)$, replication, recombination and repair $(65,8.11 \%)$, and amino acid transport and metabolism (58, 7.24\%) (Supplementary Figure 2D).

\section{Clustering and Functional Enrichment of Differentially Expressed Genes in All Treatments}

Among them, 13 DEGs were detected in all treatments, including seven upregulated and six downregulated. Some of the DEGs were involved in response to stress (GO:0006950) 

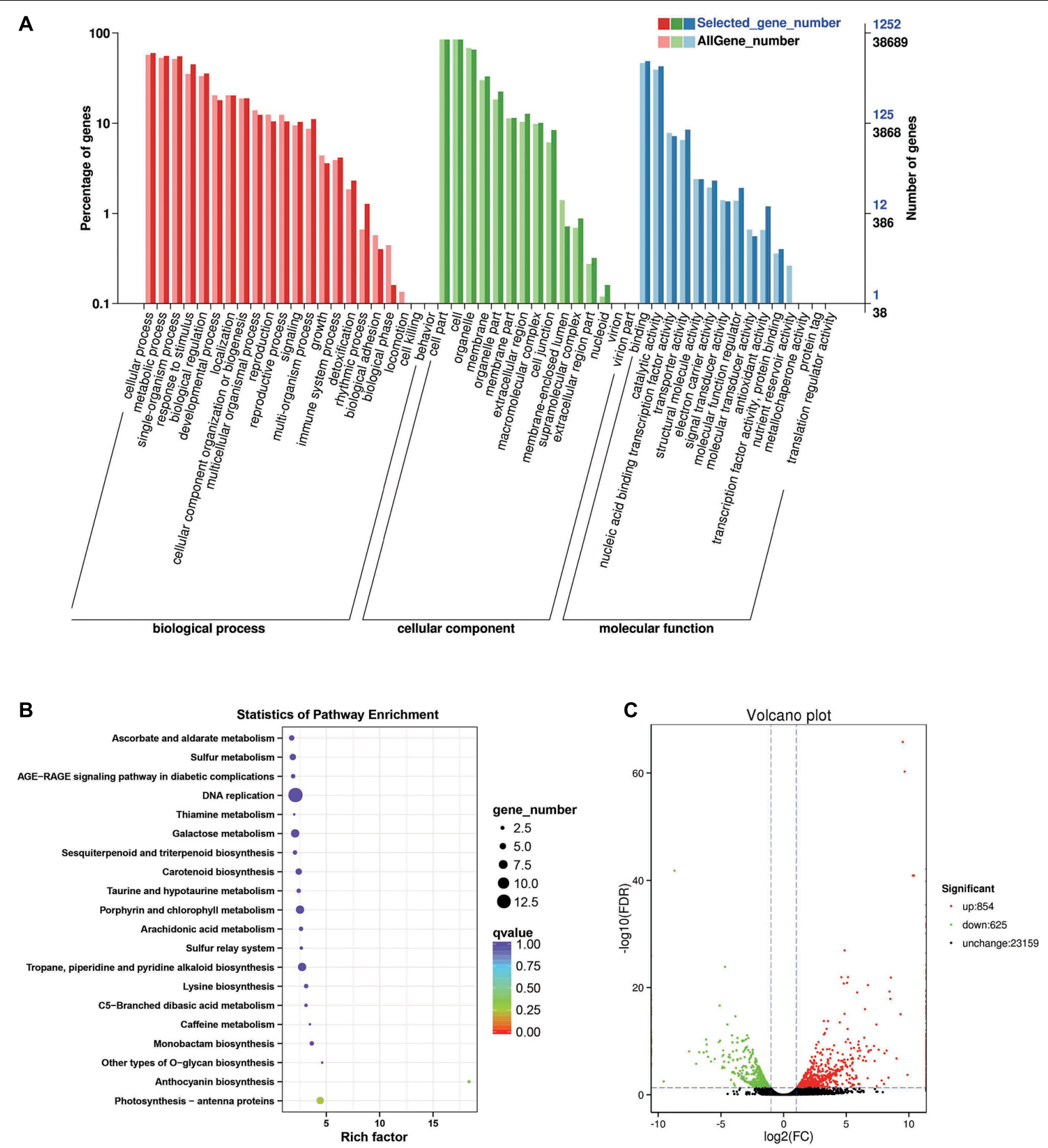

FIGURE 6 | Enrichment analysis of differentially expressed genes between C4 and C2 groups. (A) GO classification statistics of differentially expressed genes. It mainly includes three branches: biological process, cellular component, and molecular function. (B) KEGG pathway enrichment statistics of differentially expressed genes. (C) Volcano plot statistics of the number of differentially expressed genes. C2: BCL.1-4, C4: BCL.2-4.

and stimulus (GO:0050896), as well as response to abiotic stress (GO:0009628), such as freezing (GO:0050826), cold (GO:0009409), and salt (GO:0009651) stress. Some of the DEG respond to growth hormone (GO:0060416) and water deprivation (GO:0009414) (Supplementary Table 1). In addition, we performed cluster analysis on all screened differentially expressed genes (Figures 3C, 4C, 5C, 6C). Nearly all differentially expressed genes are upregulated between BcL.1-25 (N-25) and BcL.1-4 (N-4) groups; between BcL.2-25 (B-25) and BcL.2-4 (B-4) groups, most of the genes were upregulated, while the BcL.2-25 (B-25) group showed more upregulated genes; between BcL.1-25 (N-25) and BcL.2-25 (B-25) groups, the performance of upregulated genes and downregulated genes is very similar to that between BcL.1-4 (N-4) and BcL.2-4 (B-4) groups (Supplementary Figure 3). 

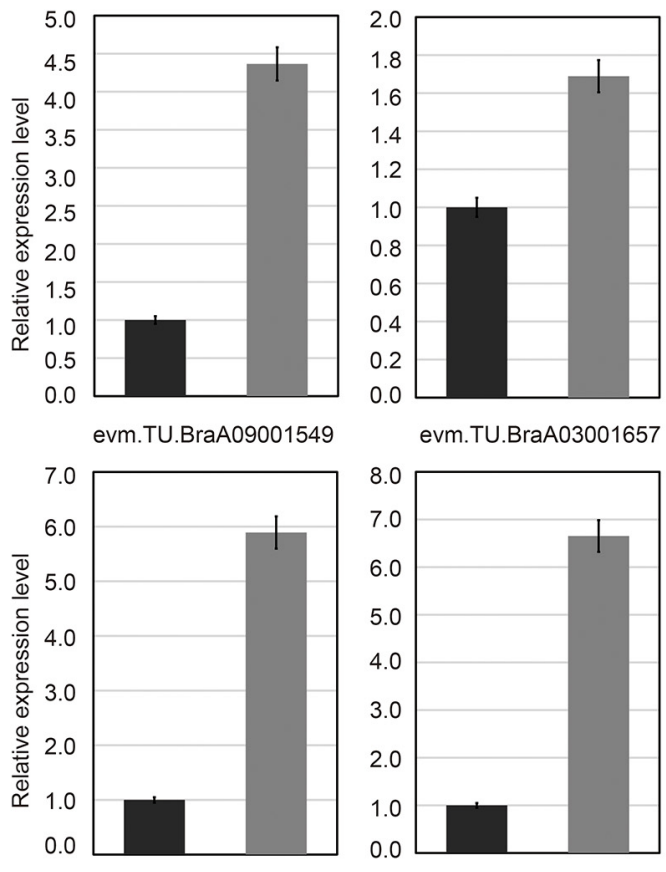

evm.TU.BraA07000744

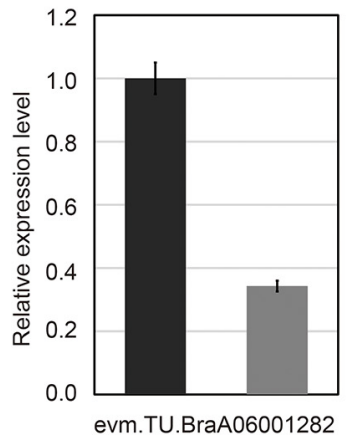

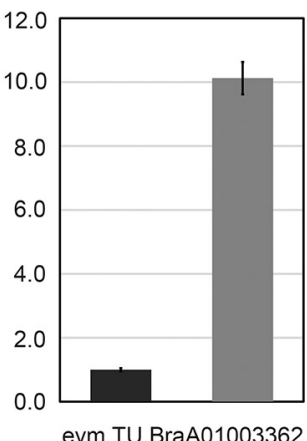

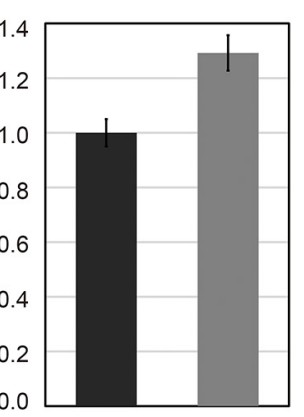

Brassica_rapa_newGene_1153

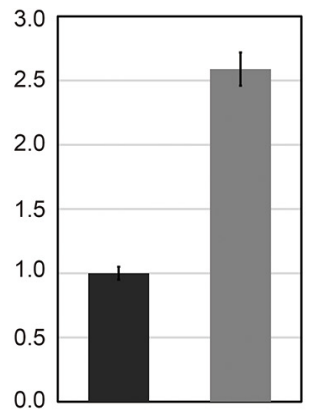

evm.TU.BraA09000675
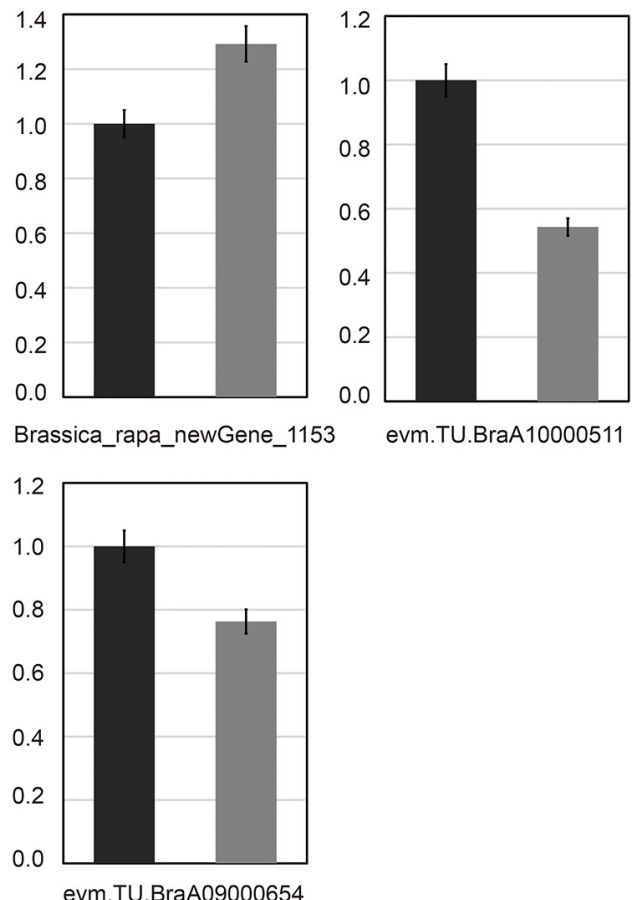

FIGURE 7 | Verification of common differentially expressed genes by quantitative real-time polymerase chain reaction (qRT-PCR). Thirteen common differentially expressed genes (DEGs) were chosen for qRT-PCR validation. The relative expression levels of each gene were expressed as the fold change between BcL.1-25 (black column) and BCL. 1-4 (gray column).

\section{Quantitative Real-Time-Polymerase Chain Reaction Validation of the Candidate Differentially Expressed Genes Responsive to Cold Tolerance}

To test the reliability of the transcriptome sequencing results, qRT-PCR analysis was used. In this study, 13 common candidate DEGs were selected and detected in all treatments by qRT-PCR analysis (Figure 7). The results of transcriptome sequencing were compared with the results of qRT-PCR experiments. Our results showed that even if the fold changes in the expression levels of certain genes detected by transcriptome sequencing and qRTPCR analysis did not match, almost all expression levels analyzed by qRT-PCR were highly consistent with the transcriptome sequencing results. These results also confirmed the reliability of transcriptome sequencing data (Figure 7). Through qRT-PCR analysis, it was found that there was only one downregulated gene (Brassica_rapa_new gene_1,153), and its expression level was different from the RNA-Seq data (Figure 7).

\section{Overview of Small RNA Sequencing Data}

In this study, these samples included C1 (BcL.1-25), C2 (BcL.1-4), C3 (BcL.2-25), and C4 (BcL.2-4), which were collected, sequenced, and analyzed. Total reads $(34,182,333)$ were generated, and 8,836,042 unique reads were isolated. After removing low-quality reads, the length distribution of the small RNAs (18-35 nt) revealed that a length of $24 \mathrm{nt}$ was the most abundant class among both clean and unique reads in all groups (Figure 8 and Table 1).

\section{Analysis of Known miRNA}

In order to obtain the details of the miRNA matched on each sample, the abovementioned reads were mapped to the 


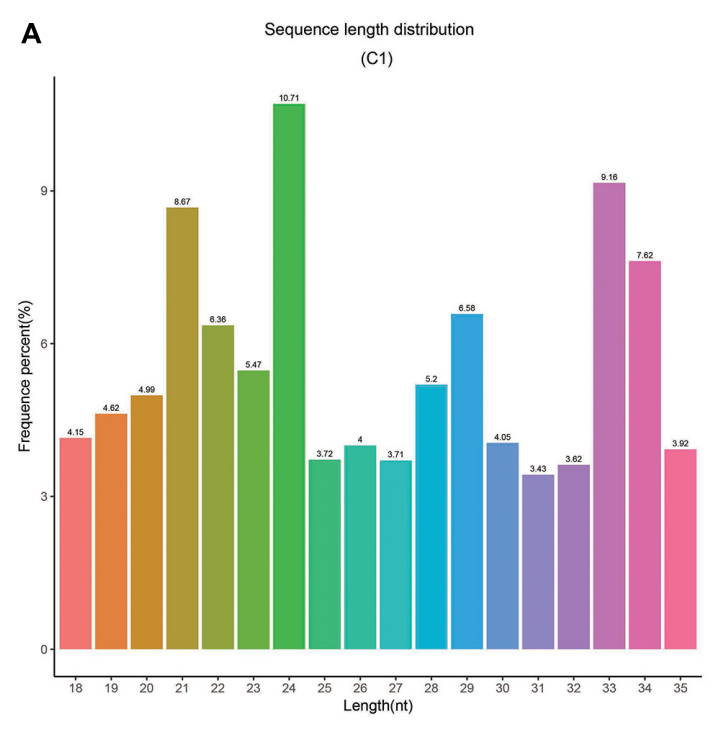

B Sequence length distribution

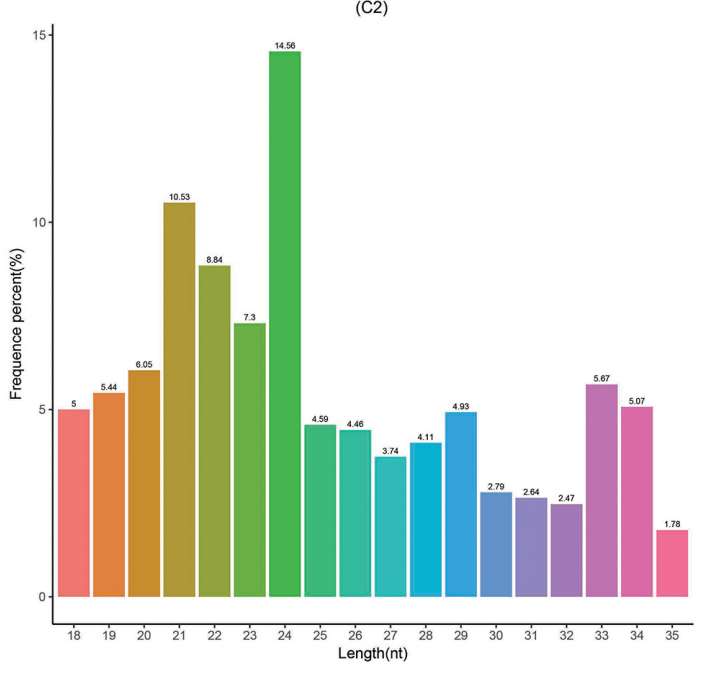

C

(C3)

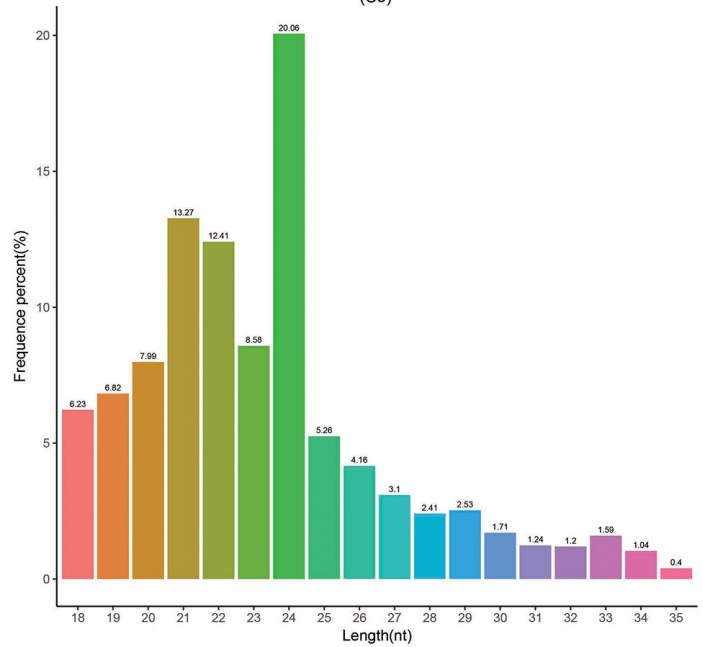

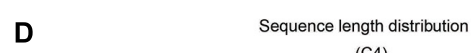

(C4)

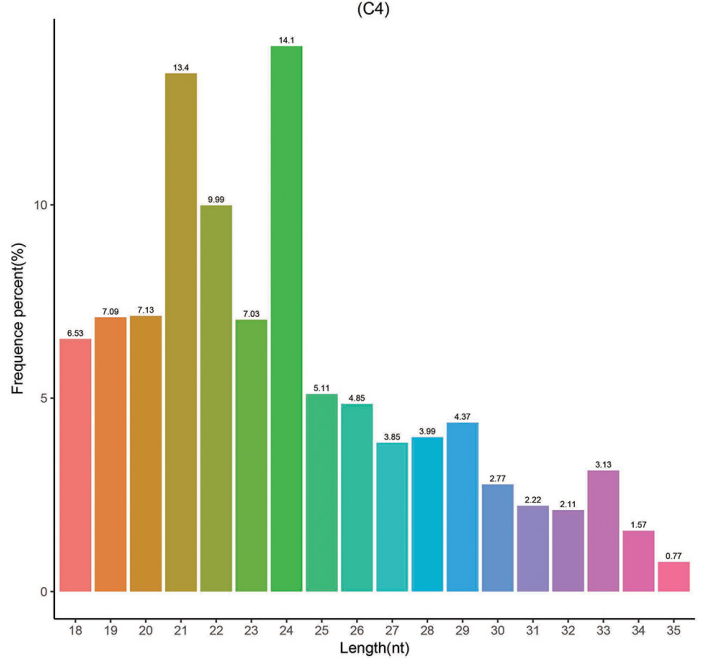

FIGURE 8 | Length distribution and abundance of small RNAs in four libraries from Pak-choi. (A) Frequence percentage (\%) and length of sRNA distribution in C1 (BCL.1-25) group. (B) Frequence percentage (\%) and length of sRNA distribution in C2 (BCL.1-4) group. (C) Frequence percentage (\%) and length of sRNA distribution in the C3 (BCL.2-25) group. (D) Frequence percentage (\%) and length of sRNA distribution in the C4 (BCL.2-4) group.

reference sequence, which are compared with the specified range of sequences in miRBase, including the secondary structure of the known miRNAs on the match, and the information on the

TABLE 1 | Type and quantity of miRNA.

\begin{tabular}{lcccc}
\hline Sample & Total reads & Total bases (bp) & Uniq reads & Uniq bases (bp) \\
\hline C1 & $7,404,323$ & $195,732,796$ & $1,708,990$ & $42,044,898$ \\
C2 & $9,762,950$ & $244,486,883$ & $2,480,460$ & $59,410,849$ \\
C3 & $8,345,217$ & $194,120,302$ & $2,669,504$ & $62,223,537$ \\
C4 & $8,669,843$ & $207,657,265$ & $1,977,088$ & $47,080,337$ \\
\hline
\end{tabular}

Sample: The sample ID; Total reads: the total number of sRNAs; Total bases (bp): The total length of the SRNA; Uniq reads: the type of SRNA; Uniq bases (bp): The total length of various SRNAs. sequence, length, and number of occurrences of the miRNA in the present invention. When the miRNA developed into a mature body from the precursor, the process was completed by dicer digestion. The specificity of the cleavage site makes the miRNA mature sequence the first base. There was a strong bias,

TABLE 2 | Known miRNA alignment table for each sample.

\begin{tabular}{lccccc}
\hline Types & Total & C1 & C2 & C3 & C4 \\
\hline Mapped mature & 110 & 78 & 91 & 95 & 91 \\
Mapped hairpin & 80 & 73 & 75 & 77 & 77 \\
Mapped uniq miRNA & 6,168 & 1,244 & 1,692 & 1,774 & 1,458 \\
Mapped total miRNA & 868,276 & 129,932 & 196,191 & 203,485 & 338,668
\end{tabular}


TABLE 3 | Statistical table of predicted new miRNA and comparison of miRNA of each sample.

\begin{tabular}{lccccc}
\hline Types & Total & C1 & C2 & C3 & C4 \\
\hline Mapped mature & 75 & 65 & 70 & 72 & 73 \\
Mapped star & 56 & 38 & 43 & 48 & 44 \\
Mapped hairpin & 84 & 78 & 80 & 81 & 81 \\
Mapped uniq miRNA & 10,967 & 2,318 & 2,837 & 2,985 & 2,827 \\
Mapped total miRNA & 346,364 & 60,344 & 80,423 & 101,934 & 103,663
\end{tabular}

so the first base distribution of miRNAs of different lengths was also carried out, in addition to the base distribution statistics of the miRNAs. As shown in Table 2, the number of miRNAs in the $\mathrm{C} 4$ group was the highest, 338,668, and the number of miRNAs in the $\mathrm{C} 1$ group was the least, 129,932. However, in the $\mathrm{C} 3$ group, the types of miRNAs were the most, 1,774;
TABLE 4 | Number of combined difference (DIFF), upregulated (UP), and downregulated (DOWN) miRNAs in each group.

\begin{tabular}{lccc}
\hline Group & Diff & Up & Down \\
\hline C2 vs. C1 & 36 & 20 & 16 \\
C4 vs. C3 & 78 & 31 & 47 \\
C3 vs. C1 & 77 & 44 & 33 \\
C4 vs. C2 & 84 & 37 & 47 \\
\hline
\end{tabular}

the $\mathrm{C} 1$ group had the least types, 1,244. In Figure 9, we listed the secondary structure of the 10 known miRNAs on the match.

\section{Predicted New miRNA}

The signature hairpin structure of miRNA precursors can be used to predict new miRNAs. As shown in Table 3, the number

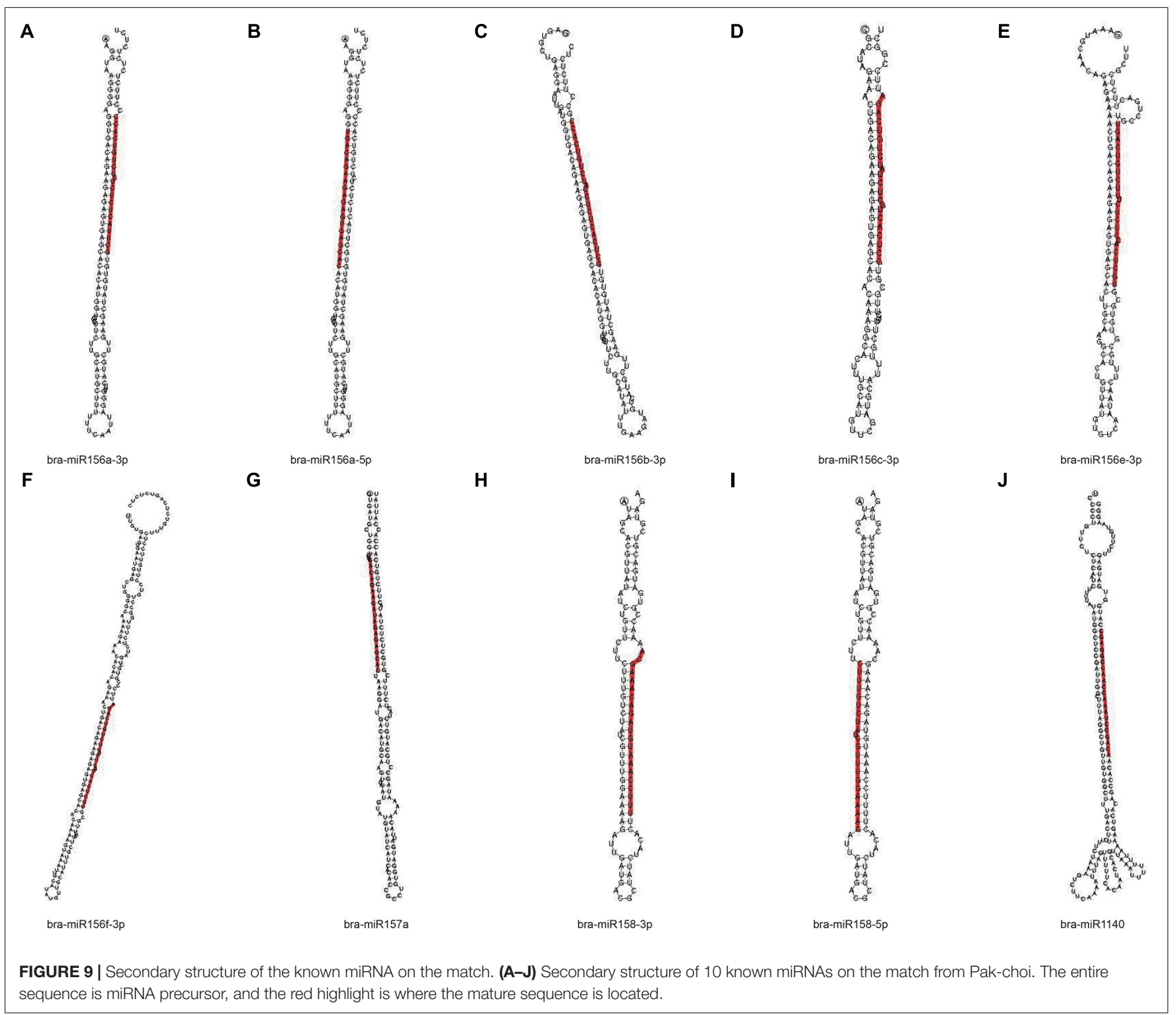


of miRNAs was the highest in the C4 group, 103,663, and the number of miRNAs was the least in the $\mathrm{C} 1$ group, 60,344. However, in the C3 group, the types of miRNAs were the most, 2,985; the $\mathrm{C} 1$ group had the least types, 2,318. We listed the secondary structure of the 10 predicted new miRNA on the match (Figure 10).

\section{Screening and Identification of Differential miRNAs}

The correlation analysis of gene expression levels between samples was carried out to test the reliability of the experimental results and the rationality of sample selection. In Figure 11C, $\mathrm{R}^{2}$, the square of the Pearson correlation coefficient, was basically at $0.772-1$, indicating that the similarity of expression patterns between samples is higher. In Figure 11, the TPM density distributions of miRNA under different experimental conditions were compared. Finally, by using volcano plots, we inferred the overall distribution of differential miRNA. Differential miRNAs were screened based on fold changes in levels and corrected significance levels (padj/q value). In the $\mathrm{C} 2$ and $\mathrm{C} 1$ groups, 20 differential miRNAs were upregulated, and 16 differential miRNAs were downregulated. In the C4 and $\mathrm{C} 3$ groups, 31 differential miRNAs were upregulated, and 47 differential miRNAs were downregulated. In the C3 and $\mathrm{C} 1$ groups, 44 differential miRNAs were upregulated, and 33 differential miRNAs were downregulated. In the $\mathrm{C} 4$ and C2 groups, 37 differential miRNAs were upregulated and 47 differential miRNAs were downregulated (Figure 12).

\section{Cluster Analysis of Differential miRNAs}

The clustering pattern of differential miRNA expression under different experimental conditions was determined by using differential miRNA cluster analysis. For each comparison combination, a set of differential miRNAs was obtained and was used for hierarchical clustering analysis. The number of miRNAs with high expression levels of $\mathrm{C} 1, \mathrm{C} 2$, and $\mathrm{C} 3$ was higher than that of the $\mathrm{C} 4$ group. In addition, the number of highly expressed miRNAs was the highest in the $\mathrm{C} 2$ group, while $\mathrm{C} 4$ had the lowest. In the $\mathrm{C} 4$ group, while some miRNAs had comparatively lower expression levels, others, such as bra-miR9557-3p and bra-miR9557-5p, were expressed at very high levels (Figure 13).

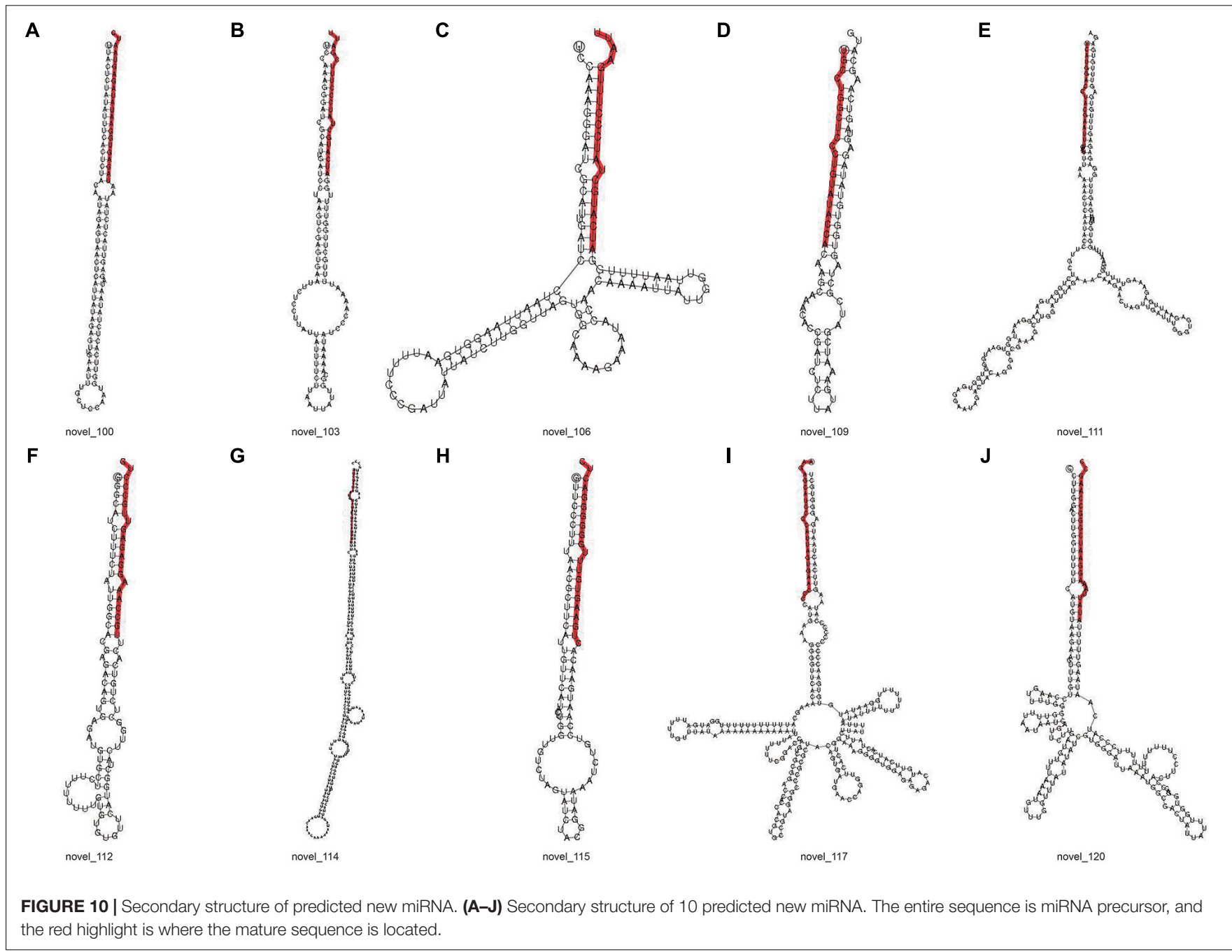



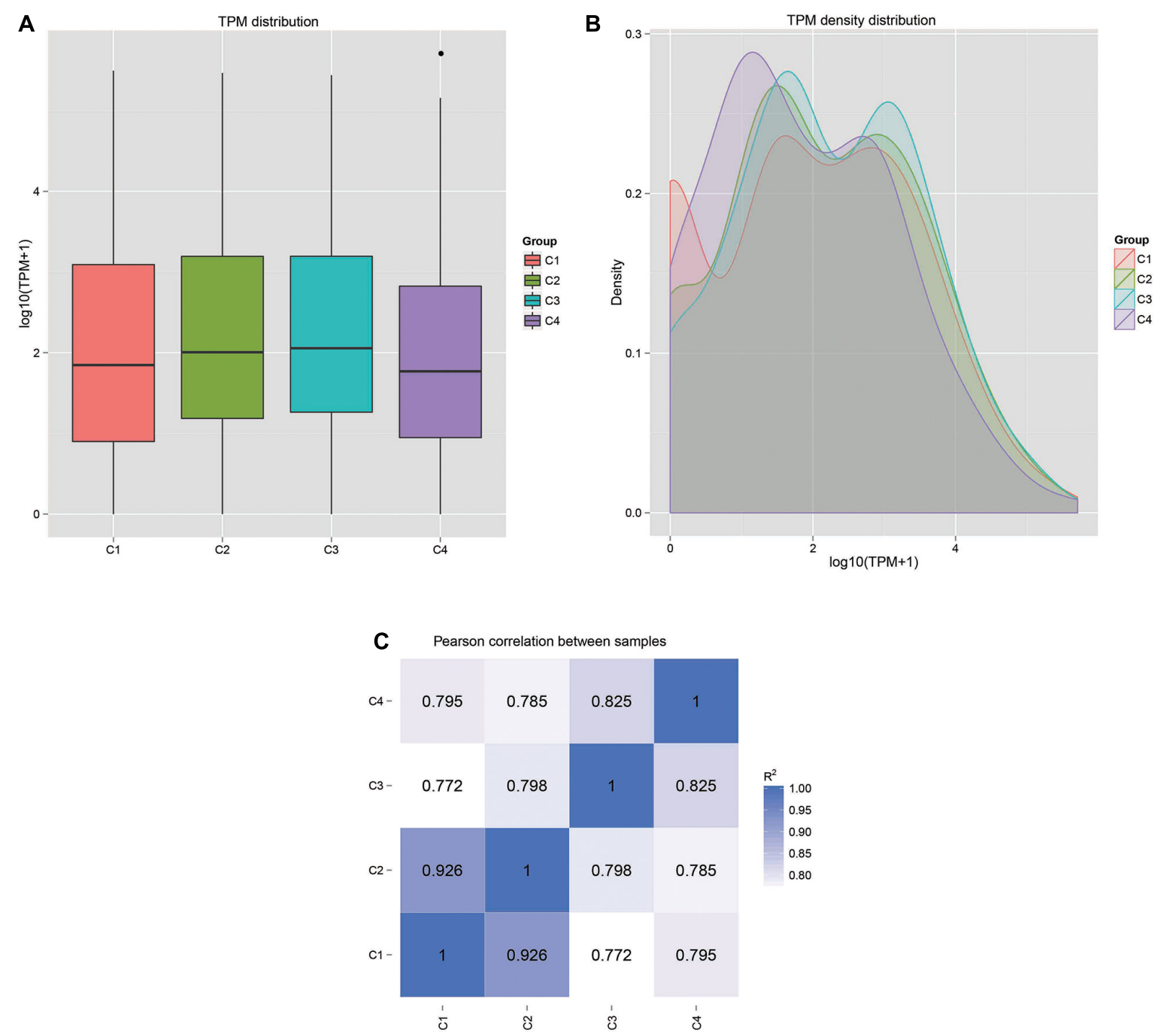

FIGURE 11 | Gene expression patterns and correlations in each sample. (A) Box plot of TPM distribution in all groups. (B) TPM density distribution diagram of miRNA expression levels. (C) Pearson correlation of miRNA expression between samples. C1: BCL.1-25, C2: BCL.1-4, C3: BCL.2-25, C4: BCL.2-4.

\section{Venn Diagram of Differential miRNAs}

Next, we show more intuitively the common and unique differences of each comparison combination. When the number of miRNAs was greater than or equal to two and less than or equal to five, the number of differential miRNAs, which was obtained by comparison in each group, can be counted and plotted as a Venn diagram (Figure 14). In Figure 14A, there were 17 common differential miRNAs; while in Figure 14B, there were 38 common differential miRNAs. In all combinations, eight common differential miRNAs are shown in Figure 14C (Table 4 and Supplementary Table 18).

\section{Enrichment Analysis of Differential miRNA Candidate Target Genes}

After obtaining the differentially expressed miRNAs between the groups, according to the correspondence between the miRNA and its target genes, we performed GO and KEGG enrichment analysis on the set of target genes of each group of differentially expressed miRNAs.

Through the GO enrichment stratification analysis, between the $\mathrm{C} 2$ and $\mathrm{C} 1$ groups, single-organism cellular process (GO:0044763, 896 genes), membrane (GO:0016020, 747 genes), and protein binding (GO:0005515, 994 genes) were the most representative GO terms in biological process, cellular component, and molecular function, respectively (Figure 15A and Supplementary Table 19). Between the C4 and C3 groups, single-organism cellular process (GO:0044763, 1,748 genes), membrane (GO:0016020, 1,431 genes), and protein binding (GO:0005515, 1,973 genes) were the most representative GO terms (Supplementary Figure 4A and Supplementary Table 21). Between the $\mathrm{C} 3$ and $\mathrm{C} 1$ groups, single-organism cellular process (GO:0044763, 1,841 genes), membrane (GO:0016020, 1,511 genes), and protein binding (GO:0005515, 2,041 genes) were 

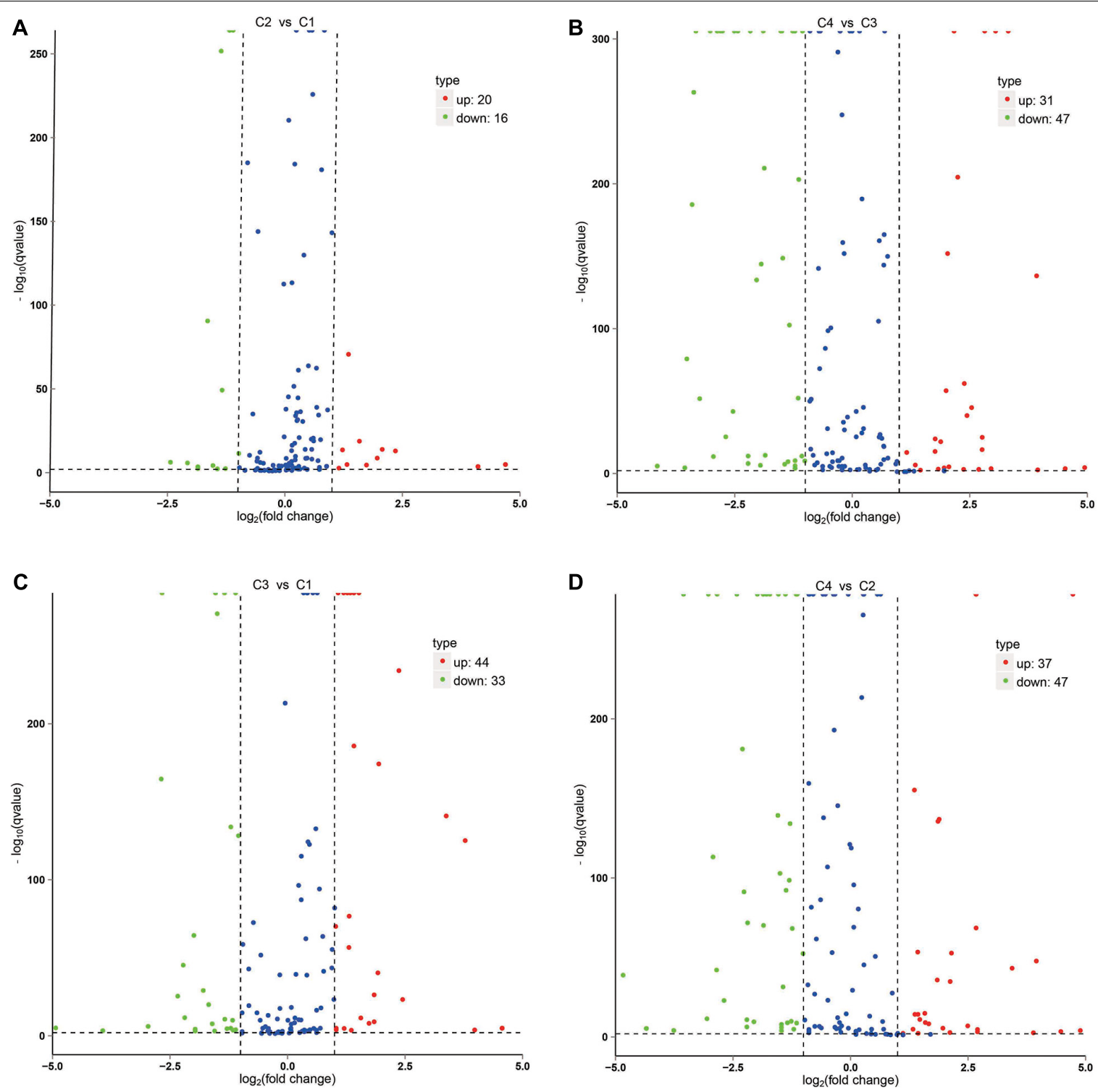

FIGURE 12 | Volcano plot of differential miRNA. (A) Differential miRNA from C2 vs. C1. (B) Differential miRNA from C4 vs. C3. (C) Differential miRNA from C3 vs. C1. (D) Differential miRNA from C4 vs. C2. $X$-axis, miRNA expression fold change in different experimental groups/different control groups; $Y$-axis, statistical significance of the change in the expression level of miRNA. Red dots indicate significantly differential upregulated miRNAs, green dots indicate significantly differential downregulated miRNAs, and blue dots indicate those without significantly differential miRNAs. C1: BcL.1-25, C2: BCL.1-4, C3: BCL.2-25, C4: BcL.2-4.

the most representative GO terms (Supplementary Figure $\mathbf{5 A}$ and Supplementary Table 23). Finally, between the C4 and C2 groups, single-organism cellular process (GO:0044763, 1,880 genes), membrane (GO:0016020, 1,515 genes), and ion binding (GO:0005515, 2,198 genes) were the most representative GO terms (Supplementary Figure 6A and Supplementary Table 25).

Through the KEGG pathway enrichment analysis, between the $\mathrm{C} 2$ and $\mathrm{C} 1$ groups, the starch and sucrose metabolism pathway was the largest, with 42 genes, followed by the plantpathogen interaction pathway, with 36 genes (Figure 15B and Supplementary Table 20). Between the C4 and C3 groups, the starch and sucrose metabolism pathway, was the largest, with 74 genes, followed by the plant-pathogen interaction pathway, with 67 genes (Supplementary Figure 4B and Supplementary Table 22). Between the C3 and C1 groups, the plant-pathogen interaction pathway, with 68 genes, and the RNA transport pathway, also containing 68 genes, are both the pathways with the most genes (Supplementary Figure 5B and Supplementary Table 24). Between the $C 4$ and $C 2$ groups, the plant-pathogen interaction pathway was the largest, with 86 genes, followed by the starch and sucrose metabolism pathway, with 78 genes (Supplementary Figure 6B and Supplementary Table 26). 


\section{Cluster analysis of differentially expressed sRNA}

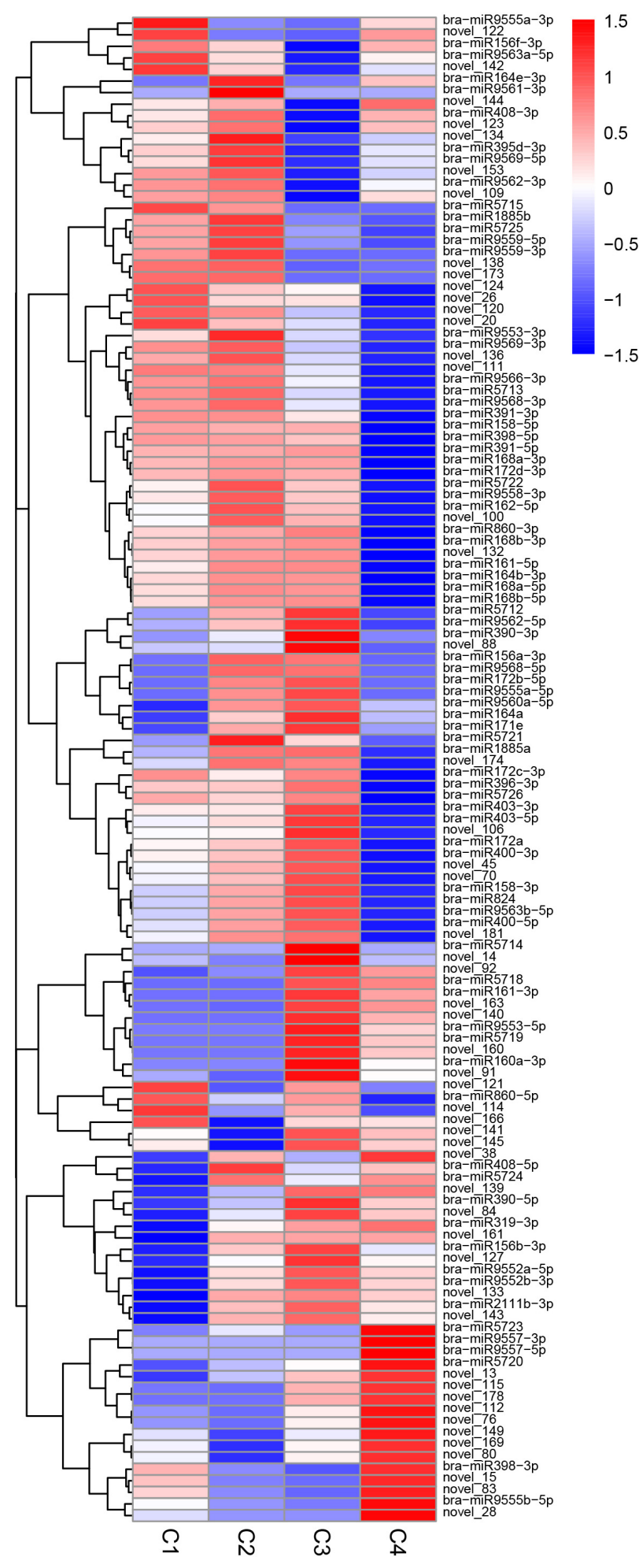

FIGURE 13 | Cluster analysis of differentially expressed miRNA. The above figure is the overall hierarchical clustering chart. Clustering is performed by the $\log _{10}($ TPM + 1) value. Red indicates high expression miRNA, and blue indicates low expression miRNA.

\section{DISCUSSION}

As a convenient tool for transcriptome analysis, RNA-Seq, has been increasingly targeted in species that do not have access to genomic sequences (Li et al., 2013; Liu et al., 2013; Soetaert et al., 2013). Here, we used RNA-Seq to measure the gene expression levels in non-heading Chinese cabbage of different varieties and under low-temperature treatment. To obtain all the DEGs from RNA-Seq data, the expression of all genes was analyzed depending on the RPKM. By comparing the data from each groups, we found that between the BcL.1-25 and BcL.14 groups, the most differentially expressed genes (6,208 DEGs) were enriched, and their number was almost six times that of the other groups, and the difference was the greatest. At the same time, the ribosome pathway is worth mentioning, and the most abundant genes (287 genes) were also shown, far more than those of the other groups. The results showed that cold stress could affect the genes involved in the expression of these pathways. Previous reports have also detected these rich pathways, partially reflecting the credibility of our results (Kreps et al., 2002; Pang et al., 2013). This also suggests that the ribosomal pathway might be involved in cold stress.

RNA-Seq was searched for some low temperature-related differentially expressed genes. qRT-PCR was used to identify gene expression levels (Taylor et al., 2010). To confirm the reliability of the RNA-Seq results, we performed qRT-PCR experiment to identify them. According to the qRT-PCR results, the expression patterns of all unigenes were consistent with the transcriptome sequencing data, showing that our experimental results were reliable.

Small RNAs are short, non-coding RNAs, usually $19-25 \mathrm{nt}$ in length, and two protruding sizes of 21 and $24 \mathrm{nt}$, respectively (Kim et al., 2012). In general, an miRNA corresponds to a 21 nt class of small molecule RNA. Research have also found that small RNAs showed a wide range of functions, including heterochromatin formation, gene silencing, and DNA methylation (Lippman and Martienssen, 2004; Penterman et al., 2007). By small RNA sequencing, the result showed that a $24-n t$ length is the most abundant category, among pure and unique reads in all groups (Figure 8). Our result was highly consistent with previous studies on A. thaliana (Rajagopalan et al., 2006), Oriza sativa (Zhu et al., 2008), Medicago truncatula (Szittya et al., 2008), and Populus trichocarpa (Puzey et al., 2012).

Furthermore, the known miRNAs are analyzed, and new miRNAs are predicted. We discovered that these selected known miRNAs have at least two stem-loop structures that are obtained by self-folding (Figures 9A-J), suggesting that there might be protein or chromosomal binding sites. Meanwhile, these predicted miRNAs have at least two stem-loop structures that are obtained by self-folding (Figures 10A-J), suggesting that there might also be protein or chromosomal binding sites. The functions of these miRNAs and their target genes were comprehensively analyzed and might provide new insights into miRNA-mediated epigenetic control of Pak-choi under low temperature.

To better understand the functional roles of these predicted miRNA targets, the target genes, which were functionally 

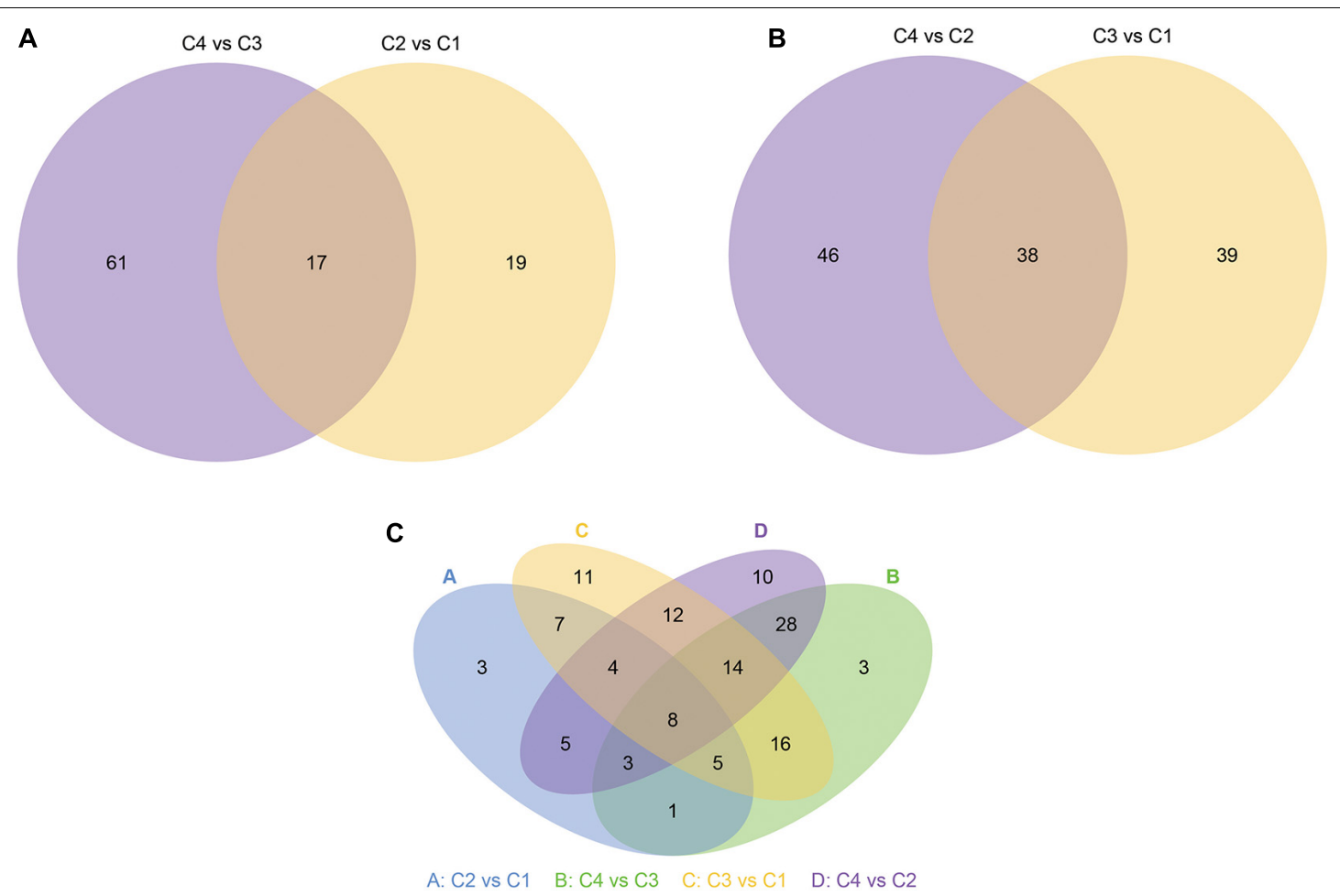

FIGURE 14 | Venn diagram of differential miRNA. The large circles represent each comparison combinations, and the sum of the number in each large circle represents the total number of differential miRNAs from the comparison combinations, and the overlapping portions of the circles represent the number of common differential miRNAs between the combinations. (A) Venn diagram of differential miRNA between C4 vs C3 and C2 vs C1 groups. (B) Venn diagram of differential miRNA between C4 vs C2 and C3 vs C1 groups. (C) Venn diagram of differential miRNA in all groups. C1: BCL.1-25, C2: BCL.1-4, C3: BCL.2-25, C4: BCL.2-4.

TABLE 5 | Primers used in the paper.

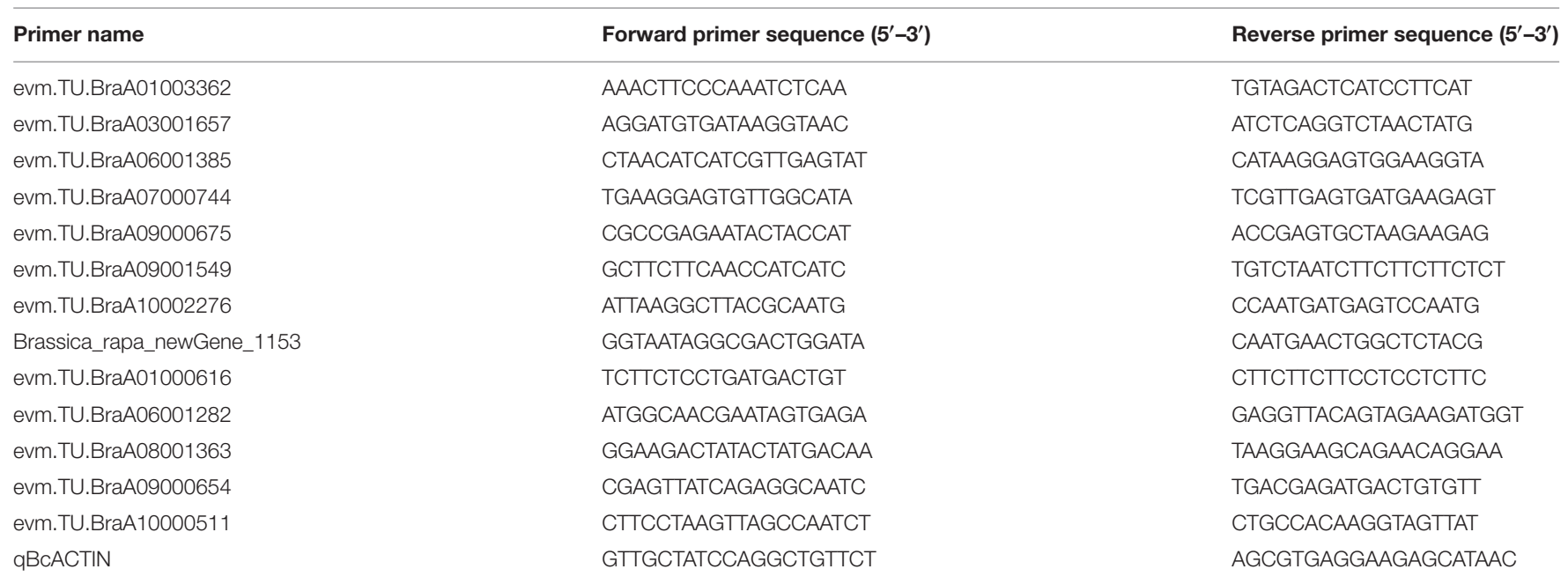

annotated in biological processes, and the KEGG pathway was also used to describe the corresponding metabolic pathways. After low-temperature treatment, most target genes were enriched in the metabolic pathways of starch and sucrose, and was basically consistent with the results of the abovementioned transcriptome study. This indicated that a series of biosynthetic and metabolic pathways might be induced after low-temperature treatment (Hu et al., 2016). Here, a combination of transcriptome and small RNA sequencing was used to analyze the cold tolerance of non-heading Chinese cabbage. This study might promote further molecular regulation mechanisms of cold tolerance in Pak-choi. 

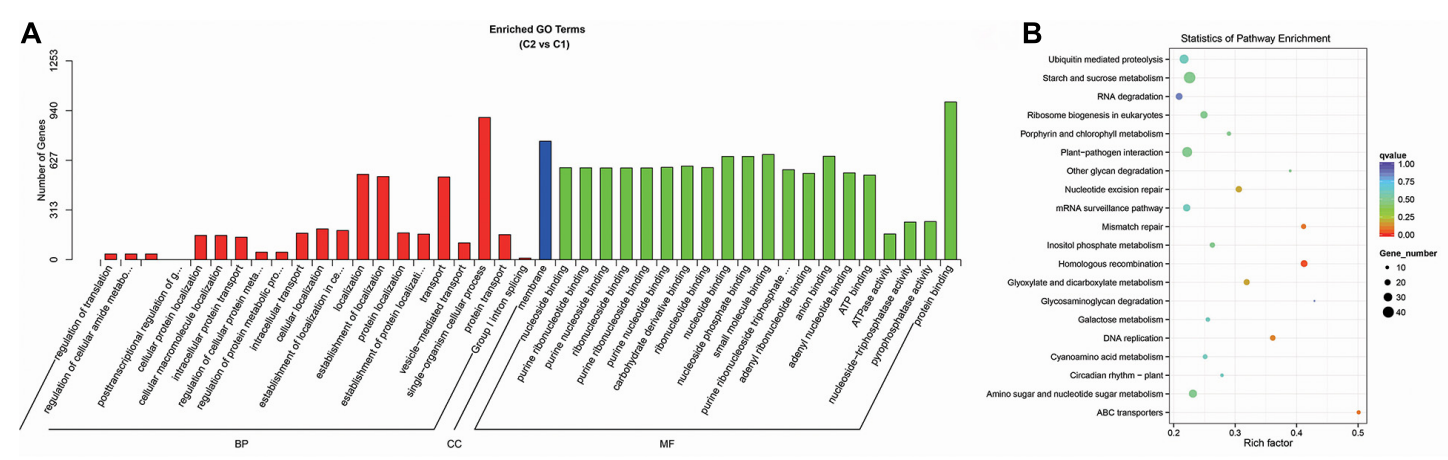

FIGURE 15 | Enrichment analysis of candidate target genes for differentially expressed miRNA between the C2 and C1 groups. (A) Histogram of GO enrichment for candidate target genes from C2 vs. C1. Three basic classifications of Go term (from left to right, BP, biological processes; CC, cellular components; MF, molecular functions). (B) KEGG enrichment scatter plot of candidate target genes from C2 vs. C1. $X$-axis, the Rich factor; $Y$-axis, the name of the pathway. The size of the dots indicates the number of candidate target genes in this pathway, and the colors of the dots correspond to different $Q$ value ranges. $C 1$ : $B C L .1-25$, $C 2$ : BCL.1-4, C3: BCL.2-25, C4: BCL.2-4.

\section{CONCLUSION}

In this study, a total of $63.43 \mathrm{~Gb}$ clean data were obtained from the transcriptome analysis. Based on the comparison results, a total of 1,860 new genes were discovered in Pak-choi, and 13 common DEGs were detected in all treatments, including seven upregulated and six downregulated. Some of the DEGs were involved in response to abiotic stresses, such as freezing, cold, and salt stresses. Among them, the cold stress response is more obvious, so we used qRT-PCR experiment to confirm changes in the expression levels of these genes. Furthermore, we performed miRNA sequencing analysis on the same material. We found that the results revealed a total of $34,182,333$ small RNA reads and a total of 88,604,604 kinds of small RNA, among which the most common size was $24 \mathrm{nt}$. In all materials, the number of common differential miRNAs was eight. The number of known mature miRNAs and the number of precursors were 110 and 80, respectively. The number of predicted novel miRNA matures, and the numbers of precursors were 75 and 84 , respectively. According to the GO and KEGG enrichment analysis, singleorganism cellular process in the biological process, membrane in the cellular component, and protein binding in the molecular function were almost all the most representative level GO terms in all data sets, while the starch and sucrose metabolism pathway was almost all the largest, followed by the plant-pathogen interaction pathway. Our findings highlighted the significance of cold signaling in Pak-choi and might provide a foundation for subsequent research under abiotic stress in the future.

\section{MATERIALS AND METHODS}

\section{Plant Growth Environment and Treatment Conditions}

The materials used in this study were non-heading Chinese cabbage varieties, Suzhouqing (BcL.1) and Sijiucaixin (BcL.2), provided by the Nanjing Agricultural University, Cabbage System Biology Laboratory. Suzhouqing (BcL.1) and Sijiucaixin (BcL.2) were both laboratory-specific and commonly used varieties, stored in an open herbarium in the laboratory. Seedlings were transferred into plastic pots containing a mixture of soil and vermiculite (volume ratio is 3:1), and cultured in a growth chamber under $16 \mathrm{~h}$ light $\left(22^{\circ} \mathrm{C}\right) / 8 \mathrm{~h}$ dark $\left(18^{\circ} \mathrm{C}\right)$. After growing for about 30 days, the plants were moved to light incubators at $4^{\circ} \mathrm{C}(B c L .1-4, B c L .2-4)$ and $25^{\circ} \mathrm{C}(B c L .1-25, B c L .2-25)$ for up to $6 \mathrm{~h}$, respectively. The leaves of plants were harvested, immediately frozen in liquid nitrogen, and stored at $-80^{\circ} \mathrm{C}$ for experimental purpose.

\section{Transcriptome Sequencing}

Following the manufacturer's instructions, we used RNAiso Plus reagent (TaKaRa, Dalian, China) to extract total RNA from the samples. RNA samples were examined by using a spectrophotometer and electrophoresed on a 1\% agarose gel. cDNA library construction and transcriptome sequencing were performed by Biomarker Technologies (Beijing, China). Transcriptome sequencing was done by Beijing Biomarker Biotechnology ${ }^{1}$ Co., Ltd. By Illumina HiSeq X 10 sequencing platform and Pe150 mode sequencing, all clean reads were subsequently mapped to the Brassica rapa reference genome sequence $(I V F C A A S v 1)^{2}$. The clean reads of each sample sequence were aligned with the designated reference genome. Gene expression analysis was performed based on the comparison results; differentially expressed genes were identified based on their expression levels in different samples, and their functional annotation and enrichment analysis were also performed (Wang et al., 2017).

\section{Transcriptome Assembly and Functional Annotation}

The raw data of the transcriptome sequencing were purified by trimming adapters, removing reads containing poly- $\mathrm{N}$, and rejecting the low-quality data (quality value $\leq 10$ or unknown

\footnotetext{
${ }^{1}$ http://wwwF.biomarker.com.cn/

${ }^{2}$ http://brassicadb.org
} 
nucleotides larger than 5\%) to get the clean reads. Meanwhile, the proportion of nucleotides with quality values greater than 30 (Q30) and GC content of the clean data were calculated. Then, all of the clean reads were assembled, using the Trinity program (Grabherr et al., 2011). First, the certain short reads with overlap regions were assembled into longer contiguous sequences for each library. Then, the distance of different contigs was recognized, mapping the clean reads, based on the paired-end information, to obtain the sequence of the transcripts. Finally, the unigenes were obtained, performing the sequence of potential transcript to the TGI Clustering tool (Pertea et al., 2003). The new genes discovered were performed with NR (Deng et al., 2006), Swiss-Prot (Apweiler et al., 2004), GO (Ashburner et al., 2000), COG (Tatusov et al., 2000), KOG (Koonin et al., 2004), Pfam (Finn et al., 2013), and KEGG (Kanehisa et al., 2004) databases, using BLAST (Altschul et al., 1997) software. KOBAS2.0 (Xie et al., 2011) was used to obtain the KEGG orthology result of the new gene for sequence alignment. After predicting the amino acid sequence of the new gene, we used the HMMER (Eddy, 1998) software to align with the Pfam database and obtained the annotation information of the new gene.

\section{Differentially Expressed Gene Analysis}

Following fragments per kilobase of exon per million fragments mapped reads (FPKM) method, the expression level of unigene was calculated (Mortazavi et al., 2008). The ratio of the FPKM values (using 0.001 instead of 0 if the FPKM was 0 ) was taken as the fold changes in the expression of each gene to identify DEGs between each groups. The false discovery rate (FDR) control method was used to identify the threshold of the p-value in multiple tests and to compute the significance of the difference in transcript abundance (Reiner et al., 2003). In this result, only fold change with $\mid \log _{2}$ (case_FPKM/control_FPKM) $\mid \geq 1$, and an FDR $\leq 0.001$ were taken as the threshold for significantly differential expression. The $\log _{2}$-transformed FPKM value for DEGs was applied to generate heat map by MeV 4.7 (Howe et al., 2011). Meanwhile, the DEGs were annotated with the GO and KEGG databases.

\section{Validation of Differentially Expressed Genes With Quantitative Real-Time Polymerase Chain Reaction}

qRT-PCR was used to confirm the expression of common differentially expressed genes in Pak-choi. Total RNA was extracted from each sample. The first-strand cDNA was synthesized, using a PrimeScript ${ }^{\mathrm{TM}}$ II First Strand cDNA synthesis kit (TaKaRa Bio, Dalian, China), according to the manufacturer's protocol. The primers were designed, using the software Beacon Designer 7.9, and listed in Table 5. The quantified expression levels of the tested genes were normalized against the housekeeping genes Cyclophilin 1 (CYP1) (Ma et al., 2016). The qRT-PCR assays were performed with three biological and technical replicates. Each reaction was performed in 20- $\mu 1$ reaction mixtures containing a diluted cDNA sample as template, SYBR Premix Ex Taq $(2 \times)$ (TaKaRa, Kyoto, Japan) and genespecific primers. Conditions for quantitative analysis were as follows: $95^{\circ} \mathrm{C}$ for $3 \mathrm{~min}$, then 40 cycles $\left(95^{\circ} \mathrm{C} 30 \mathrm{~s}, 60^{\circ} \mathrm{C} 30 \mathrm{~s}\right)$, and $72^{\circ} \mathrm{C}$ for $30 \mathrm{~s}$. qRT-PCR was performed according to a previous report (Song et al., 2016). The comparative Ct value method was adopted to analyze the relative gene expression according to a previous analysis and RNA expression levels relative to the actin gene were calculated as $2^{-\Delta \Delta C T}$ (Pfaffl, 2001; Song et al., 2016).

\section{Small RNA Sequencing}

By high-throughput sequencing (such as Illumina HiSeqTM2500/MiSeq and other sequencing platforms), sequenced raw image data files were converted into sequenced reads by base calling analysis. The raw data, containing the sequence information of read sequences and the corresponding sequencing quality information, were stored in FASTQ (abbreviated as fq) file format. Referring to the standard definition of miRNA (Allen et al., 2005; Schwab et al., 2005), the candidate target gene of miRNA was compared as the query sequence with the Brassica rapa database ${ }^{3}$. The control samples and inoculation samples were mixed for small RNA library construction, respectively. According to the reported procedures, the construction of small RNA libraries were completed (Sunkar et al., 2008). Three micrograms of total RNA per sample was used as input material for the small RNA library (Huang et al., 2018). Small RNA library construction and small RNA deep sequencing proceeded following the detailed protocol provided by the genome sequencing company (Novogene, China).

\section{Bioinformatic Analysis of Sequence Data}

The raw data were first processed through custom Perl and Python scripts. The clean data were mapped to the reference sequence in miRBase 21.0 by Bowtie (Langmead et al., 2009), without mismatch to look for known miRNAs. Then, the other reads were integrated to predict novel miRNAs using the available miREvo (Wen et al., 2010) and miRDeep2 (Friedländer et al., 2011) software. The miRNA counts as well as base bias were identified by using custom scripts. Then, the miFam.dat ${ }^{4}$ was used to look for families of known miRNAs. The novel miRNA precursor was submitted to $\mathrm{Rfam}^{5}$ to look for Rfam families.

\section{Venn Diagrams of Known miRNAs and Novel miRNAs}

Normalization formula (normalized expression = mapped read count/total reads *1,000,000) was used to estimate miRNA expression levels (Zhou et al., 2010). DEG seq R package was used to analyze the differential expression of two samples with the criterion of $\mathrm{Q}<0.01$ and $\mid \log _{2}$ (fold change) $\mid>1$ (Storey, 2003).

\section{Construction of Degradome Libraries}

Target genes of candidate miRNAs were verified by degradome sequencing by using total RNA, the same as the RNA used

\footnotetext{
${ }^{3}$ http://brassicadb.org/brad/

${ }^{4}$ http://www.mirbase.org/ftp.shtml

${ }^{5}$ http://rfam.sanger.ac.uk/search/
} 
for small RNA sequencing library construction, following the published parallel analysis of RNA End (PARE) protocol (German et al., 2009). The data analysis was processed, following the procedure instructions (Novogene, China).

\section{Target Gene Prediction and Annotation for Known and Novel miRNAs}

The psRobot_tarin psRobot was performed to predict target genes of miRNA (Wu et al., 2012). To further explore the detailed molecular mechanism of miRNAs in Pak-choi response to cold stress, the target transcripts of differentially expressed miRNAs were analyzed by GO and KEGG functional annotation suites. Subsequently, the Revigo tool $^{6}$ was implemented for enrichment analysis of the target genes. The KOBAS software was used to test the statistical enrichment of the target gene candidates in the KEGG pathways (Mao et al., 2005).

\section{DATA AVAILABILITY STATEMENT}

The full data sets have been submitted to NCBI Sequence Read Archive (SRA) under Accession SUB8670775, Bioproject: PRJNA682276.

\section{AUTHOR CONTRIBUTIONS}

JW completed the relevant experiments and wrote the manuscript. QZ, XY, and XH modified and approved the manuscript. XY and XH interpreted the results and coordinated the research. All authors have read and finalized the draft, agreeing that the manuscript will be submitted to this prestigious journal.

\section{FUNDING}

This research was funded by the Key Projects of National Key Research and Development Plan of China (2017YFD0101803), the China Agriculture Research System (CARS-23-A-06), the National Key Research and Development Plan of China (2016YFD0101701), the National Natural Science Foundation of China (NSFC) (11171155 and 11871268), and the Jiangsu Provincial Natural Science Foundation (BK20171370). The funding body supported the study, analysis of data, and writing the manuscript.

\section{ACKNOWLEDGMENTS}

This manuscript has been released as a pre-print at Research Square, doi: 10.21203/rs.2.24735/v1. The authors are grateful for the acknowledged support from the China Scholarship Council (CSC), to Biomarker Technology Co., Ltd. (Beijing,

${ }^{6}$ http://revigo.irb.hr
China), and Novogene Technology Co., Ltd. (Beijing, China) for sequencing platform.

\section{SUPPLEMENTARY MATERIAL}

The Supplementary Material for this article can be found online at: https://www.frontiersin.org/articles/10.3389/fgene.2021. 605292/full\#supplementary-material

Supplementary Figure 1 | Classification diagram of KEGG pathway types. (A) KEGG pathway types from C2 vs. C1. (B) KEGG pathway types from C4 vs. C3. (C) KEGG pathway types from C3 vs. C1. (D) KEGG pathway types from C4 vs. C2. $X$-axis, the number of genes in this pathway and their ratio to the total number of genes; $Y$-axis, the name of the KEGG metabolic pathway. $C 1$ : BCL.1-25, C2: BCL.1-4, C3: BCL.2-25, C4: BCL.2-4.

Supplementary Figure 2 | COG classification of differentially expressed genes. (A) COG classification from C2 vs. C1. (B) COG classification from C4 vs. C3. (C) COG classification from C3 vs. C1. (D) COG classification from C4 vs. C2. $X$-axis, the specific content of each COG classification, $Y$-axis, the number of genes. C1: BCL.1-25, C2: BCL.1-4, C3: BcL.2-25, C4: BcL.2-4.

Supplementary Figure 3 | Cluster analysis of differentially expressed genes. $X$-axis, name and clustering results of samples; $Y$-axis, differential gene and gene clustering results. Different columns in the figure represent different samples, and different rows represent different genes. The color represents the expression level $\log _{10}(F P K M+0.000001)$ of the gene in the sample.

Supplementary Figure 4 | Enrichment analysis of candidate target genes for differentially expressed miRNA between $\mathrm{C} 4$ and $\mathrm{C} 3$ groups. (A) Histogram of GO enrichment for candidate target genes from C4 vs. C3. Three basic classifications of Go term (from left to right, BP: biological processes, CC: cellular components, MF: molecular functions). (B) KEGG enrichment scatter plot of candidate target genes from $\mathrm{C} 4$ vs. C3. $X$-axis, the Rich factor; $Y$-axis, the name of the pathway. The size of the dots indicates the number of candidate target genes in this pathway, and the colors of the dots correspond to different $Q$-value ranges. C1: BCL.1-25, C2: BCL.1-4, C3: BCL.2-25, C4: BCL.2-4.

Supplementary Figure $\mathbf{5}$ | Enrichment analysis of candidate target genes for differentially expressed miRNA between C3 and C1 groups. (A) Histogram of GO enrichment for candidate target genes from C3 vs. C1. Three basic classifications of Go term (from left to right, BP: biological processes, CC: cellular components, MF: molecular functions). (B) KEGG enrichment scatter plot of candidate target genes from C3 vs. $\mathrm{C} 1$. $X$-axis, the Rich factor; $Y$-axis, the name of the pathway. The size of the dots indicates the number of candidate target genes in this pathway, and the colors of the dots correspond to different $\mathrm{Q}$-value ranges. C1: BCL.1-25, C2: BCL.1-4, C3: BCL.2-25, C4: BCL.2-4.

Supplementary Figure 6 | Enrichment analysis of candidate target genes for differentially expressed miRNA between $\mathrm{C} 4$ and $\mathrm{C} 2$ groups. (A) Histogram of GO enrichment for candidate target genes from C4 vs. C2. Three basic classifications of Go term (from left to right, BP: biological processes, CC: cellular components, MF: molecular functions). (B) KEGG enrichment scatter plot of candidate target genes from C4 vs. C2. $X$-axis, the Rich factor; $Y$-axis, the name of the pathway. The size of the dots indicates the number of candidate target genes in this pathway, and the colors of the dots correspond to different $Q$-value ranges. C1: BCL.1-25, C2: BCL.1-4, C3: BCL.2-25, C4: BcL.2-4.

Supplementary Table 1 | Thirteen common differentially expressed genes (seven up-regulated and six down-regulated) in all materials.

Supplementary Table 2 | GO enrichment analysis of differentially expressed genes between $\mathrm{C} 2$ and $\mathrm{C} 1$ groups.

Supplementary Table 3 | KEGG enrichment analysis of differentially expressed genes between $\mathrm{C} 2$ and $\mathrm{C} 1$ groups.

Supplementary Table 4 | GO enrichment analysis of differentially expressed genes between $\mathrm{C} 4$ and $\mathrm{C} 3$ groups. 
Supplementary Table 5 | KEGG enrichment analysis of differentially expressed genes between $\mathrm{C} 4$ and $\mathrm{C} 3$ groups.

Supplementary Table 6 | GO enrichment analysis of differentially expressed genes between $\mathrm{C} 3$ and $\mathrm{C} 1$ groups.

Supplementary Table 7 | KEGG enrichment analysis of differentially expressed genes between $\mathrm{C} 3$ and $\mathrm{C} 1$ groups.

Supplementary Table 8 | GO enrichment analysis of differentially expressed genes between $\mathrm{C} 4$ and $\mathrm{C} 2$ groups.

Supplementary Table 9 | KEGG enrichment analysis of differentially expressed genes between $\mathrm{C} 4$ and $\mathrm{C} 2$ groups.

Supplementary Table 10 | KEGG enrichment pathway type of differentially expressed genes between $\mathrm{C} 2$ and $\mathrm{C} 1$ groups.

Supplementary Table 11 | KEGG enrichment pathway type of differentially expressed genes between C4 and C3 groups.

Supplementary Table 12 | KEGG enrichment pathway type of differentially expressed genes between $\mathrm{C} 3$ and $\mathrm{C} 1$ groups.

Supplementary Table 13 | KEGG enrichment pathway type of differentially expressed genes between $\mathrm{C} 4$ and $\mathrm{C} 2$ groups.

Supplementary Table 14 | COG classification statistics of differentially expressed genes between $\mathrm{C} 2$ and $\mathrm{C} 1$ groups.

Supplementary Table 15 | COG classification statistics of differentially expressed genes between C4 and C3 groups.

\section{REFERENCES}

Abe, H., Urao, T., Ito, T., Seki, M., Shinozaki, K., and Yamaguchi-Shinozaki, K. (2003). Arabidopsis AtMYC2 (bHLH) and AtMYB2 (MYB) function as transcriptional activators in abscisic acid signaling. Plant Cell 15, 63-78. doi: $10.1105 /$ tpc.006130

Allen, E., Xie, Z., Gustafson, A. M., and Carrington, J. C. (2005). microRNAdirected phasing during trans-acting siRNA biogenesis in plants. Cell 121, 207-221. doi: 10.1016/j.cell.2005.04.004

Altschul, S. F., Madden, T. L., Schäffer, A. A., Zhang, J., Zhang, Z., Miller, W., et al. (1997). Gapped BLAST and PSI-BLAST: a new generation of protein database search programs. Nucleic Acids Res. 25, 3389-3402. doi: 10.1093/nar/25.17.3389

Anjum, S. A., Xie, X. Y., Wang, L. C., Saleem, M. F., Man, C., and Lei, W. (2011). Morphological, physiological and biochemical responses of plants to drought stress. Afr. J. Agric. Res. 6, 2026-2032.

Apweiler, R., Bairoch, A., Wu, C. H., Barker, W. C., Boeckmann, B., Ferro, S., et al. (2004). UniProt: the universal protein knowledgebase. Nucleic Acids Res. 32, D115-D119.

Ashburner, M., Ball, C. A., Blake, J. A., Botstein, D., Butler, H., Cherry, J. M., et al. (2000). Gene ontology: tool for the unification of biology. Nat. Genet. 25:25.

Ashraf, M. A., and Rahman, A. (2019). Cold stress response in Arabidopsis thaliana is mediated by GNOM ARF-GEF. Plant J. 97, 500-516. doi: 10.1111/tpj.14137

Axtell, M. J. (2013). Classification and comparison of small RNAs from plants. Annu. Rev. Plant Biol. 64, 137-159. doi: 10.1146/annurev-arplant-050312120043

Bartel, D. P. (2004). MicroRNAs: genomics, biogenesis, mechanism, and function. Cell 116, 281-297.

Chambers, C., and Shuai, B. (2009). Profiling microRNA expression in Arabidopsis pollen using microRNA array and real-time PCR. BMC Plant Biol. 9:87. doi: 10.1186/1471-2229-9-87

Cheng, Y., Liu, J., Zhang, H., Wang, J., Zhao, Y., and Geng, W. (2015). Transcriptome analysis and gene expression profiling of abortive and developing ovules during fruit development in hazelnut. PLoS One 10:e0122072. doi: 10.1371 /journal.pone. 0122072

Chinnusamy, V., Zhu, J., and Zhu, J. K. (2007). Cold stress regulation of gene expression in plants. Trends Plant Sci. 12, 444-451. doi: 10.1016/j.tplants.2007. 07.002
Supplementary Table 16 | COG classification statistics of differentially expressed genes between $\mathrm{C} 3$ and $\mathrm{C} 1$ groups.

Supplementary Table 17 | COG classification statistics of differentially expressed genes between $\mathrm{C} 4$ and $\mathrm{C} 2$ groups.

\section{Supplementary Table 18 | Number of common differential miRNAs.}

Supplementary Table 19 | GO enrichment analysis of differential miRNA target genes between $\mathrm{C} 2$ and $\mathrm{C} 1$ groups.

Supplementary Table 20 | KEGG enrichment analysis of differential miRNA target genes between $\mathrm{C} 2$ and $\mathrm{C} 1$ groups.

Supplementary Table 21 | GO enrichment analysis of differential miRNA target genes between C4 and C3 groups.

Supplementary Table 22 | KEGG enrichment analysis of differential miRNA target genes between $\mathrm{C} 4$ and $\mathrm{C} 3$ groups.

Supplementary Table 23 | GO enrichment analysis of differential miRNA target genes between $\mathrm{C} 3$ and $\mathrm{C} 1$ groups.

Supplementary Table 24 | KEGG enrichment analysis of differential miRNA target genes between $\mathrm{C} 3$ and $\mathrm{C} 1$ groups.

Supplementary Table 25 | GO enrichment analysis of differential miRNA target genes between $\mathrm{C} 4$ and $\mathrm{C} 2$ groups.

Supplementary Table 26 | KEGG enrichment analysis of differential miRNA target genes between $\mathrm{C} 4$ and $\mathrm{C} 2$ groups.

Cruz, R. P. D., and Milach, S. C. K. (2004). Cold tolerance at the germination stage of rice: methods of evaluation and characterization of genotypes. Sci. Agric. 61, 1-8. doi: 10.1590/s0103-90162004000100001

Deng, Y., Li, J., Wu, S., Zhu, Y., Chen, Y., and He, F. (2006). Integrated nr database in protein annotation system and its localization. Comput. Eng. 32, 71-72.

Devi, K., Mishra, S. K., Sahu, J., Panda, D., Modi, M. K., and Sen, P. (2016). Genome wide transcriptome profiling reveals differential gene expression in secondary metabolite pathway of Cymbopogon winterianus. Sci. Rep. 6:21026.

Dixon, R. A. (2001). Natural products and plant disease resistance. Nature 411:843. doi: $10.1038 / 35081178$

Eddy, S. R. (1998). Profile hidden Markov models. Bioinformatics 14, 755-763.

Finn, R. D., Bateman, A., Clements, J., Coggill, P., Eberhardt, R. Y., Eddy, S. R., et al. (2013). Pfam: the protein families database. Nucleic Acids Res. 42, D222-D230.

Friedländer, M. R., Mackowiak, S. D., Li, N., Chen, W., and Rajewsky, N. (2011). miRDeep2 accurately identifies known and hundreds of novel microRNA genes in seven animal clades. Nucleic Acids Res. 40, 37-52. doi: 10.1093/nar/ gkr688

Gao, J., Luo, M., Zhu, Y., He, Y., Wang, Q., and Zhang, C. (2015). Transcriptome sequencing and differential gene expression analysis in Viola yedoensis Makino (Fam. Violaceae) responsive to cadmium (Cd) pollution. Biochem. Biophys. Res. Commun. 459, 60-65. doi: 10.1016/j.bbrc.2015.02.066

Ge, G., Xiao, P., Zhang, Y., and Yang, L. (2011). The first insight into the tissue specific taxus transcriptome via Illumina second generation sequencing. PLoS One 6:e21220. doi: 10.1371/journal.pone.0021220

German, M. A., Luo, S., Schroth, G., Meyers, B. C., and Green, P. J. (2009). Construction of parallel analysis of RNA Ends (PARE) libraries for the study of cleaved miRNA targets and the RNA degradome. Nat. Protoc. 4:356. doi: $10.1038 /$ nprot. 2009.8

Goossens, A., Häkkinen, S. T., Laakso, I., Seppänen-Laakso, T., Biondi, S., De Sutter, V., et al. (2003). A functional genomics approach toward the understanding of secondary metabolism in plant cells. Proc. Natl. Acad. Sci. U.S.A. 100, 8595-8600. doi: 10.1073/pnas. 1032967100

Grabherr, M. G., Haas, B. J., Yassour, M., Levin, J. Z., Thompson, D. A., Amit, I., et al. (2011). Full-length transcriptome assembly from RNA-Seq data without a reference genome. Nat. Biotechnol. 29:644. doi: 10.1038/nbt.1883

Hamilton, J. P., and Robin Buell, C. (2012). Advances in plant genome sequencing. Plant J. 70, 177-190. doi: 10.1111/j.1365-313x.2012.04894.x 
Hao, D., Chao, M., Yin, Z., and Yu, D. (2012). Genome-wide association analysis detecting significant single nucleotide polymorphisms for chlorophyll and chlorophyll fluorescence parameters in soybean (Glycine max) landraces. Euphytica 186, 919-931. doi: 10.1007/s10681-012-0697-x

Howe, E. A., Sinha, R., Schlauch, D., and Quackenbush, J. (2011). RNA-Seq analysis in MeV. Bioinformatics 27, 3209-3210. doi: 10.1093/bioinformatics/btr490

Hu, Z., Fan, J., Xie, Y., Amombo, E., Liu, A., Gitau, M. M., et al. (2016). Comparative photosynthetic and metabolic analyses reveal mechanism of improved cold stress tolerance in bermudagrass by exogenous melatonin. Plant Physiol. Biochem. 100, 94-104. doi: 10.1016/j.plaphy.2016.01.008

Huang, F. Y., Wu, X. T., Hou, X. L., Shao, S. X., and Liu, T. K. (2018). Vernalization can regulate flowering time through microRNA mechanism in Brassica rapa. Physiol. Plant. 164, 204-215. doi: 10.1111/ppl.12692

Hussain, S., Khan, F., Cao, W., Wu, L., and Geng, M. (2016). Seed priming alters the production and detoxification of reactive oxygen intermediates in rice seedlings grown under sub-optimal temperature and nutrient supply. Front. Plant Sci. 7:439. doi: 10.3389/fpls.2016.00439

Jones-Rhoades, M. W., and Bartel, D. P. (2004). Computational identification of plant microRNAs and their targets, including a stress-induced miRNA. Mol. Cell 14, 787-799. doi: 10.1016/j.molcel.2004.05.027

Jones-Rhoades, M. W., Bartel, D. P., and Bartel, B. (2006). MicroRNAs and their regulatory roles in plants. Annu. Rev. Plant Biol. 57, 19-53. doi: 10.1146/ annurev.arplant.57.032905.105218

Kalra, S., Puniya, B. L., Kulshreshtha, D., Kumar, S., Kaur, J., Ramachandran, S., et al. (2013). De novo transcriptome sequencing reveals important molecular networks and metabolic pathways of the plant, chlorophytum borivilianum. PLoS One 8:e83336. doi: 10.1371/journal.pone.0083336

Kanehisa, M., and Goto, S. (2000). KEGG: kyoto encyclopedia of genes and genomes. Nucleic Acids Res. 28, 27-30.

Kanehisa, M., Goto, S., Kawashima, S., Okuno, Y., and Hattori, M. (2004). The KEGG resource for deciphering the genome. Nucleic Acids Res. 32, D277-D280.

Kang, H. M., and Saltveit, M. E. (2002). Chilling tolerance of maize, cucumber and rice seedling leaves and roots are differentially affected by salicylic acid. Physiol. Plant. 115, 571-576. doi: 10.1034/j.1399-3054.2002.1150411.x

Kaplan, F., Kopka, J., Haskell, D. W., Zhao, W., Schiller, K. C., Gatzke, N., et al. (2004). Exploring the temperature-stress metabolome of Arabidopsis. Plant Physiol. 136, 4159-4168. doi: 10.1104/pp.104.052142

Khraiwesh, B., Arif, M. A., Seumel, G. I., Ossowski, S., Weigel, D., Reski, R., et al. (2010). Transcriptional control of gene expression by microRNAs. Cell 140, 111-122. doi: 10.1016/j.cell.2009.12.023

Kim, B., Yu, H. J., Park, S. G., Shin, J. Y., Oh, M., Kim, N., et al. (2012). Identification and profiling of novel microRNAs in the Brassica rapa genome based on small RNA deep sequencing. BMC Plant Biol. 12:218. doi: 10.1186/1471-2229-12-218

Koonin, E. V., Fedorova, N. D., Jackson, J. D., Jacobs, A. R., Krylov, D. M., Makarova, K. S., et al. (2004). A comprehensive evolutionary classification of proteins encoded in complete eukaryotic genomes. Genome Biol. 5:R7.

Krasensky, J., and Jonak, C. (2012). Drought, salt, and temperature stress-induced metabolic rearrangements and regulatory networks. J. Exp. Bot. 63, 1593-1608. doi: $10.1093 /$ jxb/err460

Kreps, J. A., Wu, Y., Chang, H. S., Zhu, T., Wang, X., and Harper, J. F. (2002). Transcriptome changes for Arabidopsis in response to salt, osmotic, and cold stress. Plant Physiol. 130, 2129-2141. doi: 10.1104/pp.008532

Langmead, B., Trapnell, C., Pop, M., and Salzberg, S. L. (2009). Ultrafast and memory-efficient alignment of short DNA sequences to the human genome. Genome Biol. 10:R25.

Li, C., Du, C., Zeng, Y., Ma, F., Shen, Y., Xing, Z., et al. (2016). Two-dimensional visualization of nitrogen distribution in leaves of chinese cabbage (Brassica rapa subsp. Chinensis) by the fourier transform infrared photoacoustic spectroscopy technique. J. Agric. Food Chem. 64, 7696-7701. doi: 10.1021/acs.jafc.6b 03234

Li, X., Luo, J., Yan, T., Xiang, L., Jin, F., Qin, D., et al. (2013). Deep sequencingbased analysis of the Cymbidium ensifolium floral transcriptome. PLoS One 8:e85480. doi: 10.1371/journal.pone.0085480

Li, Y., Liu, G. F., Ma, L. M., Liu, T. K., Zhang, C. W., Xiao, D., et al. (2020). A chromosome-level reference genome of non-heading Chinese cabbage [Brassica campestris (syn. Brassica rapa) ssp. chinensis]. Hortic. Res. 7:212. doi: 10.1038/ s41438-020-00449-z
Li, Z., Cheng, Y., Cui, J., Zhang, P., Zhao, H., and Hu, S. (2015). Comparative transcriptome analysis reveals carbohydrate and lipid metabolism blocks in Brassica napus L. male sterility induced by the chemical hybridization agent monosulfuron ester sodium. BMC Genomics 16:206. doi: 10.1186/s12864-0151388-5

Lippman, Z., and Martienssen, R. (2004). The role of RNA interference in heterochromatic silencing. Nature 431:364. doi: 10.1038/nature 02875

Liu, Z., Ma, L., Nan, Z., and Wang, Y. (2013). Comparative transcriptional profiling provides insights into the evolution and development of the zygomorphic flower of Vicia sativa (Papilionoideae). PLoS One 8:e57338. doi: 10.1371/ journal.pone.0057338

Llave, C., Xie, Z., Kasschau, K. D., and Carrington, J. C. (2002). Cleavage of Scarecrow-like mRNA targets directed by a class of Arabidopsis miRNA. Science 297, 2053-2056. doi: 10.1126/science.1076311

Ma, R., Xu, S., Zhao, Y., Xia, B., and Wang, R. (2016). Selection and validation of appropriate reference genes for quantitative real-time PCR analysis of gene expression in Lycoris aurea. Front. Plant Sci. 7:536. doi: 10.3389/fpls.2016. 00536

Mallory, A. C., and Vaucheret, H. (2006). Functions of microRNAs and related small RNAs in plants. Nat. Genet. 38:S31.

Mao, X., Cai, T., Olyarchuk, J. G., and Wei, L. (2005). Automated genome annotation and pathway identification using the KEGG Orthology (KO) as a controlled vocabulary. Bioinformatics 21, 3787-3793. doi: 10.1093/ bioinformatics/bti430

Martin, J. A., and Wang, Z. (2011). Next-generation transcriptome assembly. Nat. Rev. Genet. 12:671. doi: 10.1038/nrg3068

Mortazavi, A., Williams, B. A., Mccue, K., Schaeffer, L., and Wold, B. (2008). Mapping and quantifying mammalian transcriptomes by RNA-Seq. Nat. Methods 5:621. doi: 10.1038/nmeth.1226

Nagalakshmi, U., Wang, Z., Waern, K., Shou, C., Raha, D., Gerstein, M., et al. (2008). The transcriptional landscape of the yeast genome defined by RNA sequencing. Science 320, 1344-1349. doi: 10.1126/science.1158441

Oliver, S. N., Dennis, E. S., and Dolferus, R. (2007). ABA regulates apoplastic sugar transport and is a potential signal for cold-induced pollen sterility in rice. Plant Cell Physiol. 48, 1319-1330. doi: 10.1093/pcp/pcm 100

Pang, T., Ye, C. Y., Xia, X., and Yin, W. (2013). De novo sequencing and transcriptome analysis of the desert shrub, Ammopiptanthus mongolicus, during cold acclimation using Illumina/Solexa. BMC Genomics 14:488. doi: 10.1186/ 1471-2164-14-488

Penterman, J., Zilberman, D., Huh, J. H., Ballinger, T., Henikoff, S., and Fischer, R. L. (2007). DNA demethylation in the Arabidopsis genome. Proc. Natl. Acad. Sci. U.S.A. 104, 6752-6757.

Pertea, G., Huang, X., Liang, F., Antonescu, V., Sultana, R., Karamycheva, S., et al. (2003). TIGR gene indices clustering tools (TGICL): a software system for fast clustering of large EST datasets. Bioinformatics 19, 651-652. doi: 10.1093/ bioinformatics/btg034

Pfaffl, M. W. (2001). A new mathematical model for relative quantification in real-time RT-PCR. Nucleic Acids Res. 29:e45.

Puzey, J. R., Karger, A., Axtell, M., and Kramer, E. M. (2012). Deep annotation of Populus trichocarpa microRNAs from diverse tissue sets. PLoS One 7:e33034. doi: 10.1371/journal.pone.0033034

Rahman, A., Kawamura, Y., Maeshima, M., Rahman, A., and Uemura, M. (2020). Plasma membrane aquaporins PIPs act in concert to regulate cold acclimation and freezing tolerance responses in Arabidopsis thaliana. Plant Cell Physiol. 61, 787-802. doi: 10.1093/pcp/pcaa005

Rajagopalan, R., Vaucheret, H., Trejo, J., and Bartel, D. P. (2006). A diverse and evolutionarily fluid set of microRNAs in Arabidopsis thaliana. Genes Dev. 20, 3407-3425. doi: 10.1101/gad.1476406

Reiner, A., Yekutieli, D., and Benjamini, Y. (2003). Identifying differentially expressed genes using false discovery rate controlling procedures. Bioinformatics 19, 368-375. doi: 10.1093/bioinformatics/btf877

Ruelland, E., Vaultier, M. N., Zachowski, A., and Hurry, V. (2009). Cold signalling and cold acclimation in plants. Adv. Bot. Res. 49, 35-150. doi: 10.1016/s00652296(08)00602-2

Salinas, J. (2002). Molecular mechanisms of signal transduction in cold acclimation. Plant Signal Transduct. 38, 116. 
Schwab, R., Palatnik, J. F., Riester, M., Schommer, C., Schmid, M., and Weigel, D. (2005). Specific effects of microRNAs on the plant transcriptome. Dev. Cell 8, 517-527. doi: 10.1016/j.devcel.2005.01.018

Shinozaki, K., and Yamaguchi-Shinozaki, K. (1996). Molecular responses to drought and cold stress. Curr. Opin.Otechnol. 7, 161-167. doi: 10.1016/s09581669(96)80007-3

Soetaert, S. S., Van Neste, C. M., Vandewoestyne, M. L., Head, S. R., Goossens, A., Van Nieuwerburgh, F. C., et al. (2013). Differential transcriptome analysis of glandular and filamentous trichomes in Artemisia annua. BMC Plant Biol. 13:220. doi: 10.1186/1471-2229-13-220

Song, X. M., Liu, G. F., Huang, Z. N., Duan, W. K., Tan, H. W., Li, Y., et al. (2016). Temperature expression patterns of genes and their coexpression with LncRNAs revealed by RNA-Seq in non-heading Chinese cabbage. BMC Genomics 17:297. doi: 10.1186/s12864-016-2625-2

Starck, Z., Niemyska, B., Bogdan, J., and Tawalbeh, R. A. (2000). Response of tomato plants to chilling stress in association with nutrient or phosphorus starvation. Plant Soil 226, 99-106.

Storey, J. D. (2003). The positive false discovery rate: a Bayesian interpretation and the q-value. Ann. Stat. 31, 2013-2035.

Sunkar, R., Kapoor, A., and Zhu, J. K. (2006). Posttranscriptional induction of two $\mathrm{Cu} / \mathrm{Zn}$ superoxide dismutase genes in Arabidopsis is mediated by downregulation of miR398 and important for oxidative stress tolerance. Plant Cell 18, 2051-2065. doi: 10.1105/tpc.106.041673

Sunkar, R., Zhou, X., Zheng, Y., Zhang, W., and Zhu, J. K. (2008). Identification of novel and candidate miRNAs in rice by high throughput sequencing. BMC Plant Biol. 8:25. doi: 10.1186/1471-2229-8-25

Szittya, G., and Burgyán, J. (2013). RNA interference-mediated intrinsic antiviral immunity in plants. Curr. Top. Microbiol. Immunol. 371, 153-181. doi: 10.1007/ 978-3-642-37765-5_6

Szittya, G., Moxon, S., Santos, D. M., Jing, R., Fevereiro, M. P., Moulton, V., et al. (2008). High-throughput sequencing of Medicago truncatula short RNAs identifies eight new miRNA families. BMC Genomics 9:593. doi: 10.1186/14712164-9-593

Tatusov, R. L., Galperin, M. Y., Natale, D. A., and Koonin, E. V. (2000). The COG database: a tool for genome-scale analysis of protein functions and evolution. Nucleic Acids Res. 28, 33-36. doi: 10.1093/nar/28.1.33

Taylor, S., Wakem, M., Dijkman, G., Alsarraj, M., and Nguyen, M. (2010). A practical approach to RT-qPCR-publishing data that conform to the MIQE guidelines. Methods 50, S1-S5.

Thakur, P., Kumar, S., Malik, J. A., Berger, J. D., and Nayyar, H. (2010). Cold stress effects on reproductive development in grain crops: an overview. Environ. Exp. Bot. 67, 429-443. doi: 10.1016/j.envexpbot.2009.09. 004

Voinnet, O. (2009). Origin, biogenesis, and activity of plant microRNAs. Cell 136, 669-687. doi: 10.1016/j.cell.2009.01.046

Wang, R., Xu, S., Wang, N., Xia, B., Jiang, Y., and Wang, R. (2017). Transcriptome analysis of secondary metabolism pathway, transcription factors, and transporters in response to methyl jasmonate in Lycoris aurea. Front. Plant Sci. 7:1971. doi: 10.3389/fpls.2016.01971

Wang, W., Chen, Q., Hussain, S., Mei, J., Dong, H., Peng, S., et al. (2016). Presowing seed treatments in direct-seeded early rice: consequences for emergence, seedling growth and associated metabolic events under chilling stress. Sci. Rep. 6:19637.

Wang, W., Vinocur, B., Shoseyov, O., and Altman, A. (2004). Role of plant heatshock proteins and molecular chaperones in the abiotic stress response. Trends Plant Sci. 9, 244-252. doi: 10.1016/j.tplants.2004.03.006
Wang, Z., Fang, B., Chen, J., Zhang, X., Luo, Z., Huang, L., et al. (2010). De novo assembly and characterization of root transcriptome using Illumina pairedend sequencing and development of cSSR markers in sweet potato (Ipomoea batatas). BMC Genomics 11:726. doi: 10.1186/1471-2164-11-726

Ward, J. A., Ponnala, L., and Weber, C. A. (2012). Strategies for transcriptome analysis in nonmodel plants. Am. J. Bot. 99, 267-276. doi: 10.3732/ajb.11 00334

Wen, H., Li, J., Song, T., Lu, M., Kan, P. Y., Lee, M. G., et al. (2010). Recognition of histone H3K4 trimethylation by the plant homeodomain of PHF2 modulates histone demethylation. J. Biol. Chem. 285, 9322-9326. doi: 10.1074/jbc.c109. 097667

Wilhelm, B. T., Marguerat, S., Watt, S., Schubert, F., Wood, V., Goodhead, I., et al. (2008). Dynamic repertoire of a eukaryotic transcriptome surveyed at single-nucleotide resolution. Nature 453:1239. doi: 10.1038/nature07002

Wu, H. J., Ma, Y. K., Chen, T., Wang, M., and Wang, X. J. (2012). PsRobot: a web-based plant small RNA meta-analysis toolbox. Nucleic Acids Res. 40, W22-W28.

Wu, S., Huang, S., Ding, J., Zhao, Y., Liang, L., Liu, T., et al. (2010). Multiple microRNAs modulate p21Cip1/Waf1 expression by directly targeting its 3' untranslated region. Oncogene 29:2302. doi: 10.1038/onc.2010.34

Xie, C., Mao, X., Huang, J., Ding, Y., Wu, J., Dong, S., et al. (2011). KOBAS 2.0: a web server for annotation and identification of enriched pathways and diseases. Nucleic Acids Res. 39, W316-W322.

Yu, S., Zhang, F., Wang, X., Zhao, X., Zhang, D., Yu, Y., et al. (2010). Genetic diversity and marker-trait associations in a collection of Pak-choi (Brassica rapa L. ssp. chinensis Makino) accessions. Genes Genomics 32, 419-428. doi: 10.1007/s13258-010-0033-6

Zhang, G., Guo, G., Hu, X., Zhang, Y., Li, Q., Li, R., et al. (2010). Deep RNA sequencing at single base-pair resolution reveals high complexity of the rice transcriptome. Genome Res. 20, 646-654. doi: 10.1101/gr.100677.109

Zhao, J., Li, S., Jiang, T., Liu, Z., Zhang, W., Jian, G., et al. (2012). Chilling stress-the key predisposing factor for causing Alternaria alternata infection and leading to cotton (Gossypium hirsutum L.) leaf senescence. PLoS One 7:e36126. doi: 10.1371/journal.pone.0036126

Zhou, L., Chen, J., Li, Z., Li, X., Hu, X., Huang, Y., et al. (2010). Integrated profiling of microRNAs and mRNAs: microRNAs located on Xq27. 3 associate with clear cell renal cell carcinoma. PLoS One 5:e15224. doi: 10.1371/journal.pone. 0015224

Zhu, J. J., Li, Y. R., and Liao, J. X. (2013). Involvement of anthocyanins in the resistance to chilling-induced oxidative stress in Saccharum officinarum L. leaves. Plant Physiol. Biochemistry 73, 427-433. doi: 10.1016/j.plaphy.2013. 07.008

Zhu, Y. G., Sun, G. X., Lei, M., Teng, M., Liu, Y. X., Chen, N. C., et al. (2008). High percentage inorganic arsenic content of mining impacted and nonimpacted Chinese rice. Environ. Sci. Technol. 42, 5008-5013. doi: 10.1021/es8001103

Conflict of Interest: The authors declare that the research was conducted in the absence of any commercial or financial relationships that could be construed as a potential conflict of interest.

Copyright (c) 2021 Wang, Zhang, You and Hou. This is an open-access article distributed under the terms of the Creative Commons Attribution License (CC BY). The use, distribution or reproduction in other forums is permitted, provided the original author(s) and the copyright owner(s) are credited and that the original publication in this journal is cited, in accordance with accepted academic practice. No use, distribution or reproduction is permitted which does not comply with these terms. 